\title{
Conditional Value-at-Risk: Semiparametric Estimation and Inference
}

\author{
Chuan-Sheng Wang and Zhibiao ZhaO* \\ Department of Statistics, Penn State University \\ University Park, PA 16802
}

\begin{abstract}
Conditional Value-at-Risk ( $\mathrm{CVaR}$ ) plays an important role in financial risk management. Nonparametric CVaR estimation suffers from the "curse of dimensionality" and slow convergence rate. To overcome these issues, we study semiparametric CVaR estimation and inference for parametric model with nonparametric noise distribution. Under a general framework that allows for many widely used time series models, we propose a semiparametric CVaR estimator that achieves the parametric convergence rate. Furthermore, to draw simultaneous inference for CVaR at multiple confidence levels, we establish a functional central limit theorem for CVaR process indexed by the confidence level and use it to study the conditional expected shortfall. A userfriendly bootstrap approach is introduced to facilitate non-expert practitioners to perform confidence interval construction for CVaR. The methodology is illustrated through both Monte Carlo studies and an application to S\&P 500 index.

JEL Codes: C14; C22; C53; G32.

Keywords: Bootstrap, Conditional expected shortfall, Conditional Value-at-Risk, Nonlinear time series, Quantile regression, Semiparametric methods.
\end{abstract}

${ }^{*}$ Corresponding author: Zhibiao Zhao. 326 Thomas Building, University Park, PA 16802, U.S.A. Tel.: 1-814-865-6552; fax: 1-814-863-7114. Email address: zuz13@stat.psu.edu. Zhao's research was supported by a NSF grant DMS-1309213 and a NIDA grant P50-DA10075-15. The content is solely the responsibility of the authors and does not necessarily represent the official views of the NIDA or the NIH. We are grateful to an Associate Editor and three anonymous referees for their very helpful comments. 


\section{Introduction}

In financial portfolio management, two most important factors of interest are the average return and its associated risk. While the average return tells the investor the mean value of the return of a particular portfolio, the risk of returns concerns the downside of the portfolio, i.e., the potentially large loss when the market moves in the opposite direction. For example, if the return takes large positive value (110\%, say) and negative value (-90\%, say) equally likely, then the average return is 10\%; however, due to the potentially large negative return, a risk-averse investor may avoid this type of double-or-none portfolio and prefer a portfolio with $5 \%$ average return but low risk. In fact, due to the importance of financial risk, financial institutions periodically monitor their risk, which forms the basis for dynamic portfolio management, to meet the supervisory guidance set by regulators.

Among many other risk measures (e.g., the standard deviation, expected shortfall, tail conditional expectation, and entropic risk measure), Value-at-Risk (hereafter, VaR) is the most prominent risk measure. For example, VaR is the widely used risk measure by regulators in banking supervision (e.g., Scaillet, 2003); also, VaR can cover the presence of netting agreements frequently found in the banking industry (e.g., Fermanian and Scaillet, 2005). For a given portfolio, its $\mathrm{VaR}$ is the threshold $L$ such that

$$
\mathbb{P}\{\text { portfolio loss during a given time period } \geq L\}=1-\tau,
$$

where $(1-\tau)$ is the confidence level, often taken to be $1 \%$ or $5 \%$. For example, at confidence level $1 \%$, the potential loss exceeds the VaR threshold $L$ with probability $1 \%$. The confidence level reflects the investor's level of tolerance of the worst scenario. A conservative investor may use a small confidence level, whereas a more aggressive investor may prefer a larger level. See Duffie and Pan (1997) and Dowd (1998) for an excellent introduction to VaR. Since (1) is derived from pure statistical inference of the underlying data-generating process, it is often termed as statistical VaR (Aït-Sahalia and Lo, 2000). To incorporate other aspects of market risk, Ait-Sahalia and Lo (2000) introduced the stateprice density based economic VaR. The state-price density is the density under which the price of any asset is the riskless-rate discounted expected payoff. Their economic VaR estimate is based on the Black-Scholes options pricing formula with a nonparametric estimate of the volatility function. In this article we focus on statistical VaR. 
Depending on the goal of the portfolio holder, another closely related VaR approach is the conditional VaR (hereafter, CVaR), which evaluates the conditional probability version of (1), conditioning on some available information. For example, an active trader may be very sensitive to short-term market information, such as the stock performance in the past week and some current global economic variables, and thus he/she may prefer the CVaR modeling, conditioning on the immediately available information when evaluating the probability in (1). We refer the reader to Chernozhukov and Umanstev (2001), Cai (2002), Fan and Gu (2003), Engle and Manganelli (2004), and Cai and Wang (2008) for various CVaR approaches. On the other hand, it may be reasonable for a retirement fund manager with a long-time vision to work under the unconditional VaR framework (1) as this marginal approach can avoid the unnecessarily volatile short-term market fluctuation. See Danielsson and de Vries (2000) for more discussions on these two approaches. In this article we focus on the CVaR approach.

To estimate VaR or CVaR, the parametric approach uses a specific parametric model (e.g., ARCH or GARCH models) with the noises following some known distribution. For example, the RiskMetrics in J. P. Morgan (1996) uses the Normal distribution; other popular choices include the Student- $t$ and some distributions that can be transformed to Normal (Hull and White, 1998). These parametric methods enjoy both nice interpretation and capability of multi-dimensional modeling, however they are subject to errors from mis-specification of the noise distribution. For example, there have been numerous discussions on whether stock returns follow Normal, Student-t, symmetric stable, or other distributions. Hypothetically, suppose returns have mean zero and variance one. From (1), at confidence level $1-\tau=1 \%$, the specification of Normal distribution would give the VaR threshold 2.33, whereas the specification of Student- $t$ distribution with 3 degrees of freedom (normalized to have variance one) would give the quite different VaR threshold 2.62. Thus, it is desirable to develop a distribution-free method.

Nonparametric VaR or CVaR estimation is a robust alternative over the parametric approach. For unconditional VaR estimation, historical simulation and its variants use empirical sample quantiles or the inverse of some marginal distribution function estimate based on the historical data to predict the future VaR; see Butler and Schachter (1998), Gourieroux, Laurent and Scaillet (2000), and Chen and Tang (2005). For CVaR, Cai (2002), Wu, Yu and Mitra (2007), and Cai and Wang (2008) proposed model-free non- 
parametric estimates based on kernel smoothing estimates of the conditional distribution function, and Cosma, Scaillet and von Sachs (2007) studied wavelets-based nonparametric estimation. As pointed out by Chen and Tang (2005), these nonparametric methods have two major advantages: (i) being distribution-free; and (ii) without imposing parametric models, such as ARCH or GARCH models. Despite their robustness to model assumptions, nonparametric approaches have some well-known challenging issues. Essentially, nonparametric CVaR methods perform estimation in a small local window of the covariates, which may contain very few or almost no observations for high-dimensional covariates. This is the well-known "curse of dimensionality" issue. Other practically challenging issues include bandwidth selection and slow convergence rate. See Li and Racine (2007) for discussions.

This article has two main contributions. Our first contribution is to propose a semiparametric CVaR estimator and establish its $\sqrt{n}$ asymptotic normality. As discussed above, both the parametric and nonparametric approaches have their strengths and weaknesses, and we propose combining their strengths via a semiparametric approach of parametric model with nonparametric noise distribution. Our semiparametric approach has several appealing features. First, the parametric model structure is capable of modeling the dependence of returns on high-dimensional covariates. This can avoid the "curse of dimensionality" issue of the nonparametric approach. Furthermore, the parametric component has the advantage of including some non-Markovian behavior (e.g., GARCH) as opposed to a pure nonparametric kernel regression approach. Second, adopting nonparametric noise distribution can avoid the error from distributional mis-specification. Third, unlike the ARCH/GARCH VaR modeling, our methodology is developed under a very general framework that allows for many linear and nonlinear processes. Fourth, the proposed CVaR estimator can achieve the parametric $\sqrt{n}$ convergence rate.

Despite the vast literature on VaR and CVaR estimation, little attention has been paid to calculating the standard error of the estimates; Chen and Tang (2005) studied this problem for unconditional VaR estimation. Our second contribution is to develop methodology for statistical inference of CVaR. First, we provide consistent standard error for the semiparametric CVaR estimator, which is useful in confidence interval construction. Second, to draw simultaneous inference for CVaR at multiple confidence levels, we establish a functional central limit theorem (hereafter, CLT). As an application of the functional CLT, we study semiparametric estimation of conditional expected shortfall (hereafter, CES). Third, 
to facilitate non-expert practitioners to construct confidence intervals for CVaR and CES, we introduce an easy-to-implement bootstrap approach. One major advantage of the bootstrap approach is that practitioners can choose their own particular model and parameter estimation method to address semiparametric CVaR and CES inference.

The article is organized as follows. Section 2 contains main results on semiparametric CVaR estimation, asymptotic normality, and standard error calculation. Section 3 studies CVaR process and an application to CES. Section 4 introduces bootstrap inference. Section 5 briefly studies semiparametric conditional distribution estimation. Numerical analysis is presented in Sections 6 and 7. Finally, technical conditions and proofs are in Section 8.

For a matrix $A=\left(a_{i, j}\right)$, write $|A|=\left(\sum_{i, j} a_{i, j}^{2}\right)^{1 / 2}$. For a random vector $\mathbf{Z}$, write $\mathbf{Z} \in \mathcal{L}^{q}, q>0$, if $\mathbb{E}\left(|\mathbf{Z}|^{q}\right)<\infty$. Throughout, $\stackrel{p}{\rightarrow}$ stands for convergence in probability.

\section{Semiparametric CVaR Estimation}

Let $Y_{i} \in \mathbb{R}$ be scalar-valued portfolio loss (i.e., negative gain) at time $i$. Suppose the loss $Y_{i}$ depends on some $p$-dimensional covariates $\mathbf{X}_{i} \in \mathbb{R}^{1 \times p}$. In practice, the covariates $\mathbf{X}_{i}$ may include both the historical market information, such as the past portfolio loss $Y_{i-1}, \ldots, Y_{i-q}$, and some overall exogenous economic variables $\mathbf{U}_{i} \in \mathbb{R}^{p-q}$, such as the inflation rates and unemployment rates. Similar to (1), at confidence level $(1-\tau)$, the CVaR of $Y_{i}$ given $\mathbf{X}_{i}=x$, denoted by $\operatorname{CVaR}(1-\tau \mid x)$, is defined as

$$
\operatorname{CVaR}(1-\tau \mid x)=L \quad \text { such that } \quad \mathbb{P}\left\{Y_{i} \geq L \mid \mathbf{X}_{i}=x\right\}=1-\tau
$$

Therefore, conditioning on $\mathbf{X}_{i}=x$, the loss $Y_{i}$ exceeds $\operatorname{CVaR}(1-\tau \mid x)$ with probability $(1-\tau)$. In particular, if $\mathbf{X}_{i}=\left(Y_{i-1}, \ldots, Y_{i-p}\right)$, then CVaR $(1-\tau \mid x)$ is the predicted CVaR of $Y_{i}$ given $Y_{i-1}, \ldots, Y_{i-p}$. Our goal is to estimate and make inference about CVaR $(1-\tau \mid x)$.

Denote by $Q(\tau \mid x):=Q\left(\tau \mid \mathbf{X}_{i}=x\right)$ the conditional $\tau$-th quantile of $Y_{i}$ given $\mathbf{X}_{i}=x$. From (2), $\operatorname{CVaR}(1-\tau \mid x)=Q(\tau \mid x)$. From now on we shall focus on $Q(\tau \mid x)$.

Remark 1. In this paper we focus on the case of fixed level $1-\tau$, even though it may be very small. An alternative approach is the extreme quantile approach, i.e., $1-\tau \rightarrow 0$ as sample size $n \rightarrow \infty$ so that in (2) the threshold $L \rightarrow \infty$. If we consider aggregated monthly returns as the sum of daily returns, then the moderate deviation approach in $\mathrm{Wu}$ 
and Zhao (2008) may be applied here, but the asymptotic theory is more challenging. This is beyond the scope of the current paper and will serve as a direction for future research.

\subsection{The nonparametric quantile regression approach}

The conditional $\tau$-th quantile $Q(\tau \mid x)$ can be estimated by local linear quantile regression

$$
\tilde{Q}(\tau \mid x)=\hat{a}_{0}, \quad\left(\hat{a}_{0}, \hat{a}_{1}\right)=\underset{a_{0} \in \mathbb{R}, a_{1} \in \mathbb{R}^{p}}{\operatorname{argmin}} \sum_{i=1}^{n} \rho_{\tau}\left\{Y_{i}-a_{0}-\left(\mathbf{X}_{i}-x\right) a_{1}\right\} K\left(\frac{\mathbf{X}_{i}-x}{b_{n}}\right),
$$

where $\rho_{\tau}(v)=v\left(\tau-\mathbf{1}_{v \leq 0}\right)$ is the check function, $K(\cdot)$ is a $p$-variate kernel function, and $b_{n}>0$ is bandwidth; see $\mathrm{Yu}$ and Jones (1997). Another nonparametric quantile estimation approach is based on the inverse of conditional distribution function estimate (Cai, 2002; Wu, Yu and Mitra, 2007; Cai and Wang, 2008). See Chapter 6 in Li and Racine (2007).

While nonparametric quantile regression is robust against model structure, it also suffers from several drawbacks. First, due to the "curse of dimensionality", it is generally infeasible to perform nonparametric estimation for $p \geq 3$. As a result, when predicting VaR based on historical loss, nonparametric approach can use only very local recent historical information ( $p=1$ or 2 at most). Second, $p$-dimensional nonparametric conditional quantile estimation has convergence rate $\sqrt{n b_{n}^{p}}$, which can be quite slow as $b_{n} \rightarrow 0$. Third, it is a practically non-trivial issue to select the bandwidth $b_{n}$ (Li and Racine, 2007).

\subsection{The proposed semiparametric approach}

By Section 2.1, nonparametric quantile estimation is not very appealing in CVaR estimation. In this section we propose a semiparametric approach. Specifically, we assume

$$
Y_{i}=G\left(\theta, \varepsilon_{i}, \mathbf{X}_{i}\right)
$$

where $G(\theta, \varepsilon, x)$ is a parametric function with unknown $k$-dimensional parameter $\theta \in \mathbb{R}^{k}$, and $\left\{\varepsilon_{i}\right\}_{i \in \mathbb{Z}}$ are unobservable i.i.d. noises that may represent unobserved heterogeneity or technological shocks. We leave the distribution of $\varepsilon_{i}$ completely unspecified, leading to a semiparametric structure. This semiparametric approach can avoid potential misspecification on the distribution of $\varepsilon_{i}$. For example, a normal distribution may perform poorly in the presence of Cauchy distributed noises. On the other hand, the parametric 
assumption on $G(\theta, \cdot, \cdot)$ allows us to avoid the "curse of dimensionality" in nonparametric approach and thus has the capability of high-dimensional CVaR estimation.

Model (4) assumes that the function $G$ can be well parameterized by some parameter $\theta$ with the distribution of $\varepsilon_{i}$ unspecified. In applications, we can use a two-stage procedure to determine the function form $G$. In the first stage, we may use the sieve nonparametric estimation method (e.g., Chen, 2007) or nonparametric kernel estimation method (e.g., Matzkin, 2003) to estimate the function nonparametrically. In the second stage, we then check whether the estimated nonparametric function can be parameterized by some existing models. The parametric specification stage is equally important in all aspects of parametric modeling in the literature, such as model fitting and forecasting, in addition to VaR risk management. Since our goal is not to develop new specification testing method but to develop CVaR estimation for given model, in this paper we assume that the researcher has decided a specific model prior to CVaR estimation. In fact, almost all existing works on parametric VaR (e.g., EWMA in RiskMetrics of J.P. Morgan, 1994; robust-EWMA in Guermat and Harris, 2001; CAViaR in Engle and Manganelli, 2004; skewed-EWMA in Gerlach, Lu and Huang, 2013) also took the same approach. Furthermore, the effect from model mis-specification will be examined through simulations in Section 6.

Example 1. (Nonlinear AR models) Let $\mathbf{X}_{i}=\left(Y_{i-1}, \ldots, Y_{i-p}\right)$, then (4) becomes

$$
Y_{i}=G\left(\theta, \varepsilon_{i}, Y_{i-1}, \ldots, Y_{i-p}\right),
$$

a nonlinear autoregressive (AR) model of order $p$. An important special case of (5) is the class of nonlinear ARCH model

$$
Y_{i}=\mu\left(\theta, Y_{i-1}, \ldots, Y_{i-p}\right)+\sigma\left(\theta, Y_{i-1}, \ldots, Y_{i-p}\right) \varepsilon_{i}
$$

for parametric functions $\mu(\theta, \cdot)$ and $\sigma(\theta, \cdot)>0$ with unknown parameter $\theta$. Model (6) includes many popular nonlinear models; see Fan and Yao (2003).

Example 2. (Nonlinear ARX models) A more flexible generalization of (5) is the nonlinear AR with exogenous/external inputs $(\mathrm{ARX})$ model $\left(\mathbf{X}_{i}=\left(Y_{i-1}, \ldots, Y_{i-q}, \mathbf{U}_{i}\right)\right)$ :

$$
Y_{i}=G\left(\theta, \varepsilon_{i}, Y_{i-1}, \ldots, Y_{i-q}, \mathbf{U}_{i}\right)
$$

where $\mathbf{U}_{i} \in \mathbb{R}^{1 \times(p-q)}$ are exogenous or external variables. For example, an exogenous variable can be the inflation rates or unemployment rates affecting stock returns $Y_{i}$. The 
classical linear ARX model is $Y_{i}=\sum_{j=1}^{q} \phi_{j} Y_{i-j}+\mathbf{U}_{i} \beta+\varepsilon_{i}$ for coefficients $\phi_{1}, \ldots, \phi_{q} \in$ $\mathbb{R}, \beta \in \mathbb{R}^{p-q}$. Model (7) allows flexible nonlinear generalization. For example, (7) includes the ARCH model with exogenous inputs:

$$
Y_{i}=\sum_{j=1}^{q} \phi_{j} Y_{i-j}+\mathbf{U}_{i} \beta+\varepsilon_{i}\left(\alpha_{0}^{2}+\sum_{j=1}^{q} \alpha_{j}^{2} Y_{i-j}^{2}+\mathbf{U}_{i}^{2} \gamma^{2}\right)^{1 / 2}, \quad \beta, \gamma \in \mathbb{R}^{p-q} .
$$

This model generalizes Engle's ARCH model to allow for exogenous variables.

Example 3. (Nonlinear GARCH models) Consider the nonlinear GARCH model

$$
Y_{i}=\sigma_{i} \varepsilon_{i} \quad \text { with } \quad \sigma_{i}=g\left(\theta, \sigma_{i-1}, \ldots, \sigma_{i-q}, Y_{i-1}, \ldots, Y_{i-r}\right),
$$

for a parametric function $g>0$. By specifying different forms of $g$, this general model includes many widely used variants of GARCH models, including the classical GARCH model (Bollerslev, 1986), the EGARCH model (Nelson, 1991), the GJR-GARCH model (Glosten, Jagannathan and Runkle, 1993), and the TGARCH model (Zakoian, 1994), just to name a few. Under appropriate conditions (e.g., Wu and Shao, 2004), by recursive iteration, $\sigma_{i}$ admits the representation $\sigma_{i}=g^{*}\left(\theta, Y_{i-1}, Y_{i-2}, \ldots\right)$ for some function $g^{*}$. Thus, (8) becomes the $\mathrm{ARCH}(\infty)$ model $Y_{i}=g^{*}\left(\theta, Y_{i-1}, Y_{i-2}, \ldots\right) \varepsilon_{i}$ and we can take the covariates $\mathbf{X}_{i}=\left(Y_{i-1}, Y_{i-2}, \ldots\right)$. In practice, we need not to know the function form $g^{*}$; instead we can recursively compute $\sigma_{i}=g\left(\theta, \sigma_{i-1}, \ldots, \sigma_{i-q}, Y_{i-1}, \ldots, Y_{i-r}\right)$ when the parameter $\theta$ is known or can be estimated. Since the GARCH model (8) is non-Markovian, the "curse of dimensionality" of $g^{*}$ makes it infeasible to use the nonparametric approach in Section 2.1 to estimate $Q\left(\tau \mid \mathbf{X}_{i}=x\right)$.

To motivate our semiparametric estimator of $Q(\tau \mid x):=Q\left(\tau \mid \mathbf{X}_{i}=x\right)$, we assume that in (4) the function $G\left(\theta, \varepsilon_{i}, \mathbf{X}_{i}\right)$ is strictly increasing in $\varepsilon_{i}$ and that $\varepsilon_{i}$ is independent of $\mathbf{X}_{i}$. By definition, given $\mathbf{X}_{i}=x, Q(\tau \mid x)$ is the $\tau$-th quantile of $Y_{i}=G\left(\theta, \varepsilon_{i}, \mathbf{X}_{i}\right)=G\left(\theta, \varepsilon_{i}, x\right)$. Note that the $\tau$-th quantile of any strictly increasing transformation of a random variable is the same transformation of the $\tau$-th quantile of that random variable. Therefore,

$$
Q(\tau \mid x)=\tau \text {-th quantile of } G\left(\theta, \varepsilon_{i}, x\right)=G\left(\theta, Q_{\varepsilon}(\tau), x\right),
$$

where $Q_{\varepsilon}(\tau)$ is the $\tau$-th quantile function of $\varepsilon_{i}$. In practice, both $\theta$ and $Q_{\varepsilon}(\tau)$ are unknown, and we propose estimating $Q(\tau \mid x)$ by plugging some consistent estimates of $\theta$ and $Q_{\varepsilon}(\tau)$ 
into (9). However, the true innovations $\left\{\varepsilon_{i}\right\}$ are not observable. Fortunately, under the above strictly increasing assumption on $G\left(\theta, \varepsilon_{i}, \mathbf{X}_{i}\right)$ (as a function $\varepsilon_{i}$ ), we can invert the function to obtain $\varepsilon_{i}$. Formally, we impose Assumption 1 below.

Assumption 1. For any given $\left(\theta, \mathbf{X}_{i}\right)$, the function $G\left(\theta, \varepsilon_{i}, \mathbf{X}_{i}\right)$ is strictly increasing in $\varepsilon_{i}$ so the inverse $G^{-1}\left(\theta, \cdot, \mathbf{X}_{i}\right)$ exists and

$$
\varepsilon_{i}=H\left(\theta, Y_{i}, \mathbf{X}_{i}\right) \quad \text { with } \quad H\left(\theta, Y_{i}, \mathbf{X}_{i}\right)=G^{-1}\left(\theta, Y_{i}, \mathbf{X}_{i}\right) . \quad \text { (the inverse) }
$$

Assumption 1 is satisfied for many practical models. In fact, in the context of nonparametric estimation of non-additive functions, Matzkin (2003) imposed the same condition. Clearly, Assumption 1 is satisfied for the nonlinear ARCH model in (6) and the nonlinear GARCH model in (8). In addition, it is satisfied for some transformation models. Let $\Lambda(\cdot)$ be a strictly increasing transformation function. Then Assumption 1 is satisfied for the model $\Lambda\left(Y_{i}\right)=\mathbf{X}_{i} \theta+\varepsilon_{i}$ or equivalently $Y_{i}=\Lambda^{-1}\left(\mathbf{X}_{i} \theta+\varepsilon_{i}\right)$. The latter model includes the well-known Box-Cox transformation and all the transformation models studied in Horowitz (1996). Under appropriate conditions on the conditional hazard condition, duration models with unobserved heterogeneity also satisfy Assumption 1; we refer the reader to Matzkin (2003) for more details.

Under Assumption 1, in view of (9), we propose the following estimation procedure:

(i) Let $\hat{\theta}$ be a consistent estimate of $\theta$. From (10), we can estimate the innovation $\varepsilon_{i}$ by

$$
\text { generalized residuals: } \quad \hat{\varepsilon}_{i}=H\left(\hat{\theta}, Y_{i}, \mathbf{X}_{i}\right) \text {. }
$$

(ii) Estimate $Q_{\varepsilon}(\tau)$ by $\hat{Q}_{\varepsilon}(\tau)$, the sample $\tau$-th quantile of $\left\{\hat{\varepsilon}_{i}\right\}$. Formally,

$$
\hat{Q}_{\varepsilon}(\tau)=\inf \left\{z: \hat{F}_{\varepsilon}(z) \geq \tau\right\}, \quad \text { where } \quad \hat{F}_{\varepsilon}(z)=\frac{1}{n} \sum_{i=1}^{n} \mathbf{1}_{\hat{\varepsilon}_{i} \leq z} .
$$

(iii) Plugging $\hat{\theta}$ and $\hat{Q}_{\varepsilon}(\tau)$ into (9), we propose the following estimator:

$$
\hat{Q}(\tau \mid x)=G\left(\hat{\theta}, \hat{Q}_{\varepsilon}(\tau), x\right) .
$$

Compared with the nonparametric quantile estimation approach in Section 2.1, the proposed semiparametric estimator (13) is easy to implement and does not require any 
bandwidth. To derive the asymptotic normality, a key step is to study the residual empirical process $\hat{F}_{\varepsilon}(z)$ in (12). In fact, due to the important applications in model diagnostics and hypothesis testing, the topic of residual empirical process itself has attracted much attention; see, e.g., Lee and Wei (1999) and Horváth and Teyssière (2001) for residual empirical process from AR models and ARCH models, respectively. Theorem 1 below establishes the uniform approximation of the generalized-residual empirical process. Given the general form of model (4), our result is more general than existing ones.

Denote by $F_{\varepsilon}(\cdot), f_{\varepsilon}(\cdot)$, and $Q_{\varepsilon}(\cdot)$, respectively, the distribution, density, and quantile functions of $\varepsilon_{i}$. Throughout we assume that $G(\theta, \varepsilon, x)$ is continuously differentiable in $\theta$ and $\varepsilon$, with corresponding partial derivatives $\dot{G}_{\theta}(\theta, \varepsilon, x)$ and $\dot{G}_{\varepsilon}(\theta, \varepsilon, x)$.

Theorem 1. Recall $\hat{F}_{\varepsilon}(z)$ in (12). Suppose that Assumption 1 and Assumptions 3-4 (in the Appendix) hold. Further assume that $\hat{\theta}=\theta+O_{p}\left(n^{-1 / 2}\right)$. Then for any given $c>0$,

$$
\sup _{|z| \leq c}\left|\hat{F}_{\varepsilon}(z)-\frac{1}{n} \sum_{i=1}^{n} \mathbf{1}_{\varepsilon_{i} \leq z}-f_{\varepsilon}(z) \mathbb{E}\left[\frac{\dot{G}_{\theta}\left(\theta, z, \mathbf{X}_{0}\right)}{\dot{G}_{\varepsilon}\left(\theta, z, \mathbf{X}_{0}\right)}\right]^{T}(\hat{\theta}-\theta)\right|=o_{p}\left(n^{-1 / 2}\right) .
$$

Here and hereafter $\mathbf{X}_{0}$ has the same distribution as $\mathbf{X}_{i}$.

By Theorem 1, the asymptotic expansion of $\hat{F}_{\varepsilon}(z)$ has two components: the first term $n^{-1} \sum_{i=1}^{n} \mathbf{1}_{\varepsilon_{i} \leq z}$ is the empirical process of the true noises $\left\{\varepsilon_{i}\right\}$, and the second term $f_{\varepsilon}(z) \mathbb{E}\left[\dot{G}_{\theta}\left(\theta, z, \mathbf{X}_{0}\right) / \dot{G}_{\varepsilon}\left(\theta, z, \mathbf{X}_{0}\right)\right]^{T}(\hat{\theta}-\theta)$ is the bias correction term due to the estimation error of $\hat{\theta}$. Therefore, in order to derive the asymptotic distribution of $\hat{Q}(\tau \mid x)$, it is necessary to impose some condition on $\hat{\theta}-\theta$.

Assumption 2. The estimator $\hat{\theta} \in \mathbb{R}^{k}$ of $\theta \in \mathbb{R}^{k}$ admits the Bahadur-type representation

$$
\hat{\theta}-\theta=\frac{1}{n} \sum_{i=1}^{n} D\left(\theta, \varepsilon_{i}, \mathbf{X}_{i}\right)+o_{p}\left(n^{-1 / 2}\right),
$$

for some $D(\theta, \cdot, \cdot) \in \mathbb{R}^{k}$ satisfying $D\left(\theta, \varepsilon_{i}, \mathbf{X}_{i}\right) \in \mathcal{L}^{2}$ and $\mathbb{E}\left[D\left(\theta, \varepsilon_{i}, \mathbf{X}_{i}\right) \mid \mathbf{X}_{i}\right]=0$.

Assumption 2 asserts that $\hat{\theta}-\theta$ has a linear leading term plus some negligible error. This type of Bahadur representations has been established for different models in the literature. For example, Hall and Yao (2003) obtained Bahadur representation of quasi-maximum likelihood estimates for ARCH and GARCH models, and Zhao (2010) established Bahadur representation for pseudo-likelihood estimate of stochastic regression models. See Section 2.4 below for more discussions. 
Theorem 2. Suppose that Assumption 1 and Assumptions $3-5$ in Section 8.1 hold.

(i) If $\hat{\theta}=\theta+O_{p}\left(n^{-1 / 2}\right)$, then $\hat{Q}(\tau \mid x)$ is $\sqrt{n}$-consistent, i.e., $\hat{Q}(\tau \mid x)=Q(\tau \mid x)+O_{p}\left(n^{-1 / 2}\right)$.

(ii) If in addition Assumption 2 holds (recall $D\left(\theta, \varepsilon_{i}, \mathbf{X}_{i}\right)$ there), then the CLT holds

$$
\sqrt{n}[\hat{Q}(\tau \mid x)-Q(\tau \mid x)] \Rightarrow N(0, \Gamma(\tau)),
$$

where $\Gamma(\tau)=\dot{G}_{\varepsilon}\left(\theta, Q_{\varepsilon}(\tau), x\right)^{2} \mathbb{E}\left[W_{1}(\tau)^{2}\right]$ and

$$
W_{i}(\tau)=\frac{\tau-\mathbf{1}_{\varepsilon_{i}<Q_{\varepsilon}(\tau)}}{f_{\varepsilon}\left(Q_{\varepsilon}(\tau)\right)}+\left\{\frac{\dot{G}_{\theta}\left(\theta, Q_{\varepsilon}(\tau), x\right)}{\dot{G}_{\varepsilon}\left(\theta, Q_{\varepsilon}(\tau), x\right)}-\mathbb{E}\left[\frac{\dot{G}_{\theta}\left(\theta, Q_{\varepsilon}(\tau), \mathbf{X}_{0}\right)}{\dot{G}_{\varepsilon}\left(\theta, Q_{\varepsilon}(\tau), \mathbf{X}_{0}\right)}\right]\right\}^{T} D\left(\theta, \varepsilon_{i}, \mathbf{X}_{i}\right)
$$

By Theorem 2, the proposed semiparametric CVaR estimator can achieve $\sqrt{n}$ parametric convergence rate, regardless of the dimensionality of the covariates $\mathbf{X}_{i}$. By contrast, for practical reason, the nonparametric quantile regression approach in (3) works only for $p=1$ or 2 and has convergence rate $\sqrt{n b_{n}^{p}}$ for some non-trivial choice of bandwidth $b_{n}$.

Denote by $\tilde{Q}_{\varepsilon}(\tau)$ the sample quantile of the true innovations $\left\{\varepsilon_{i}\right\}$. By the well-known theory for sample quantiles, $\sqrt{n}\left[\tilde{Q}_{\varepsilon}(\tau)-Q_{\varepsilon}(\tau)\right] \Rightarrow N\left(0, \tau(1-\tau) / f_{\varepsilon}\left(Q_{\varepsilon}(\tau)\right)^{2}\right)$. Note that $\mathbb{E}\left\{\left[\left(\tau-\mathbf{1}_{\varepsilon_{i}<Q_{\varepsilon}(\tau)}\right) / f_{\varepsilon}\left(Q_{\varepsilon}(\tau)\right)\right]^{2}\right\}=\tau(1-\tau) / f_{\varepsilon}\left(Q_{\varepsilon}(\tau)\right)^{2}$. Thus, the first term of $W_{i}(\tau)$ in (17) reflects the variation of the sample quantile of the true innovations $\left\{\varepsilon_{i}\right\}$. On the other hand, the second term of $W_{i}(\tau)$ reflects the error due to the estimator $\hat{\theta}$. The first term is an intrinsic feature of sample quantiles, which never vanishes; the second term generally does not vanish but may vanish under some special settings. For example, if $G\left(\theta, \varepsilon_{i}, \mathbf{X}_{i}\right) \equiv$

$G\left(\varepsilon_{i}, \mathbf{X}_{i}\right)$ is completely known (does not depend on any parameter), then $\dot{G}_{\theta}(\theta, \cdot, \cdot)=0$ and consequently the second term vanishes. As a second example, if $G\left(\theta, \varepsilon_{i}, \mathbf{X}_{i}\right) \equiv G\left(\theta, \varepsilon_{i}\right)$ does not depend on $\mathbf{X}_{i}$, then the second term also vanishes. Intuitively, in the latter case, $\left\{Y_{i}\right\}$ are i.i.d. and therefore $Q(\tau \mid x)$ is simply the marginal quantile of $\left\{Y_{i}\right\}$.

\subsection{Consistent estimate of the limiting variance}

In the vast literature on VaR and CVaR estimation, the standard error calculation has been largely ignored. Chen and Tang (2005) studied this problem for unconditional VaR estimation; for nonparametric CVaR estimation in Cai and Wang (2008), they did not provide consistent estimate for the standard error, and any such attempt would involve nonparametric function estimation with properly chosen bandwidth. Here we consider consistent estimate of the limiting variance $\Gamma(\tau)$ in (16). We propose the following procedure: 
(i) Using the estimated innovations $\left\{\hat{\varepsilon}_{i}\right\}$ in (11), we estimate the density $f_{\varepsilon}(z)$ of $\varepsilon_{i}$ by

$$
\hat{f}_{\varepsilon}(z)=\frac{1}{n h_{n}} \sum_{i=1}^{n} K\left(\frac{\hat{\varepsilon}_{i}-z}{h_{n}}\right)
$$

where $h_{n}>0$ is the bandwidth. For example, the rule-of-thumb bandwidth choice (Silverman, 1986) is $h_{n}=0.9 n^{-1 / 5} \min \left\{\operatorname{sd}\left(\hat{\varepsilon}_{i}\right), \operatorname{IQR}\left(\hat{\varepsilon}_{i}\right) / 1.34\right\}$, where $\operatorname{sd}\left(\hat{\varepsilon}_{i}\right)$ and $\operatorname{IQR}\left(\hat{\varepsilon}_{i}\right)$ are the sample standard deviation and sample interquartile of $\left\{\hat{\varepsilon}_{i}\right\}$.

(ii) Plugging $\hat{\varepsilon}_{i}, \hat{f}_{\varepsilon}, \hat{\theta}, \hat{Q}_{\varepsilon}(\tau)$ [see $\left.(12)\right]$ into $W_{i}(\tau)$ in (17) to obtain the sample version

$$
\widehat{W}_{i}(\tau)=\frac{\tau-\mathbf{1}_{\hat{\varepsilon}_{i}<\hat{Q}_{\varepsilon}(\tau)}}{\hat{f}_{\varepsilon}\left(\hat{Q}_{\varepsilon}(\tau)\right)}+\left\{\frac{\dot{G}_{\theta}\left(\hat{\theta}, \hat{Q}_{\varepsilon}(\tau), x\right)}{\dot{G}_{\varepsilon}\left(\hat{\theta}, \hat{Q}_{\varepsilon}(\tau), x\right)}-\frac{1}{n} \sum_{i=1}^{n} \frac{\dot{G}_{\theta}\left(\hat{\theta}, \hat{Q}_{\varepsilon}(\tau), \mathbf{X}_{i}\right)}{\dot{G}_{\varepsilon}\left(\hat{\theta}, \hat{Q}_{\varepsilon}(\tau), \mathbf{X}_{i}\right)}\right\}^{T} D\left(\hat{\theta}, \hat{\varepsilon}_{i}, \mathbf{X}_{i}\right) .
$$

(iii) Using the sample variance of $\widehat{W}_{i}(\tau)$ to estimate $\Gamma(\tau)$ by

$$
\hat{\Gamma}(\tau)=\dot{G}_{\varepsilon}\left(\hat{\theta}, \hat{Q}_{\varepsilon}(\tau), x\right)^{2} \frac{1}{n-1} \sum_{i=1}^{n}\left[\widehat{W}_{i}(\tau)-\bar{W}(\tau)\right]^{2}, \quad \bar{W}(\tau)=\frac{1}{n} \sum_{i=1}^{n} \widehat{W}_{i}(\tau)
$$

Theorem 3. Suppose that Assumptions 1-2 and Assumptions 3-6 in Section 8.1 hold. In (18), assume that: (i) the kernel $K(\cdot)$ has bounded support and bounded derivative; and (ii) the bandwidth $h_{n}$ satisfies $n h_{n}^{4} \rightarrow \infty$. Recall $\Gamma(\tau)$ defined in (16). Then

$$
\hat{\Gamma}(\tau) \stackrel{p}{\rightarrow} \Gamma(\tau)
$$

Consequently, from Theorem 2,

$$
\frac{\sqrt{n}[\hat{Q}(\tau \mid x)-Q(\tau \mid x)]}{\sqrt{\hat{\Gamma}(\tau)}} \Rightarrow N(0,1)
$$

By Theorem 3, an asymptotic $(1-\alpha)$ confidence interval for $Q(\tau \mid x)$ is

$$
\hat{Q}(\tau \mid x) \pm q_{1-\alpha}\left(\frac{\hat{\Gamma}(\tau)}{n}\right)^{1 / 2}
$$

where $q_{1-\alpha}$ is the $(1-\alpha)$ quantile of $|N(0,1)|$. In Section 4 below we introduce an alternative bootstrap approach that can bypass the estimation of $\Gamma(\tau)$. 


\subsection{Bahadur representation in Assumption 2}

From Theorem 2 , the $\sqrt{n}$-consistency requires only $\hat{\theta}=\theta+O_{p}\left(n^{-1 / 2}\right)$, but the CLT relies on the Bahadur representation (15) in Assumption 2. Also, the variance estimator $\hat{\Gamma}(\tau)$ in (19) relies on the Bahadur representation. Such Bahadur representation depends on the specific model structure and parameter estimation method. We briefly discuss this issue.

An important example of (4) is the nonlinear model with heteroscedastic errors:

$$
Y_{i}=\mu\left(\theta, \mathbf{X}_{i}\right)+\sigma\left(\theta, \mathbf{X}_{i}\right) \varepsilon_{i}
$$

for i.i.d. noises $\left\{\varepsilon_{i}\right\}$ with $\mathbb{E}\left(\varepsilon_{i}\right)=0$ and $\mathbb{E}\left(\varepsilon_{i}^{2}\right)=1$ and parametric functions $\mu(\theta, \cdot)$ and $\sigma(\theta, \cdot)>0$. Model (22) satisfies Assumption 1. Consider the pseudo-likelihood estimate:

$$
\hat{\theta}=\underset{\theta}{\operatorname{argmin}} \sum_{i=1}^{n}\left\{\left[\frac{Y_{i}-\mu\left(\theta, \mathbf{X}_{i}\right)}{\sigma\left(\theta, \mathbf{X}_{i}\right)}\right]^{2}+2 \log \sigma\left(\theta, \mathbf{X}_{i}\right)\right\} .
$$

Theorem 2 in Zhao (2010) established the Bahadur representation (15) with

$$
D\left(\theta, \varepsilon_{i}, \mathbf{X}_{i}\right)=\mathcal{I}(\theta)^{-1}\left[\frac{\varepsilon_{i} \dot{\mu}\left(\theta, \mathbf{X}_{i}\right)}{\sigma\left(\theta, \mathbf{X}_{i}\right)}+\left(\varepsilon_{i}^{2}-1\right) \frac{\dot{\sigma}\left(\theta, \mathbf{X}_{i}\right)}{\sigma\left(\theta, \mathbf{X}_{i}\right)}\right],
$$

where $\dot{\mu}\left(\theta, \mathbf{X}_{i}\right)$ and $\dot{\sigma}\left(\theta, \mathbf{X}_{i}\right)$ are the partial derivatives with respect to $\theta$, and

$$
\mathcal{I}(\theta)=\mathbb{E}\left[\frac{\dot{\mu}\left(\theta, \mathbf{X}_{0}\right) \dot{\mu}\left(\theta, \mathbf{X}_{0}\right)^{T}+2 \dot{\sigma}\left(\theta, \mathbf{X}_{0}\right) \dot{\sigma}\left(\theta, \mathbf{X}_{0}\right)^{T}}{\sigma^{2}\left(\theta, \mathbf{X}_{0}\right)}\right] .
$$

Other estimation methods lead to different Bahadur representations, depending on specific loss functions. For maximum likelihood estimation, the Bahadur representation depends on the score function. For quantile regression based estimator, consider the special case of $(4)$ that $\sigma(\cdot, \cdot) \equiv 1$ and $Q_{\varepsilon}(0.5)=0\left(\varepsilon_{i}\right.$ has median zero), then the median quantile regression estimator is the minimizer of $\sum_{i=1}^{n}\left|Y_{i}-\mu\left(\theta, \mathbf{X}_{i}\right)\right|$, which satisfies (15) with

$$
D\left(\theta, \varepsilon_{i}, \mathbf{X}_{i}\right)=\left\{\mathbb{E}\left[\dot{\mu}\left(\theta, \mathbf{X}_{0}\right) \dot{\mu}\left(\theta, \mathbf{X}_{0}\right)^{T}\right]\right\}^{-1} \frac{\dot{\mu}\left(\theta, \mathbf{X}_{i}\right)}{f_{\varepsilon}(0)}\left(\frac{1}{2}-\mathbf{1}_{\varepsilon_{i}<0}\right) .
$$

See Jurečková and Procházka (1994). Zhao and Xiao (2014) obtained a Bahadur representation for quantile regression estimator of the location-scale model $Y_{i}=\mathbf{X}_{i} \beta+\left(\mathbf{X}_{i} \gamma\right) \varepsilon_{i}$. He and Shao (1996) obtained Bahadur representations for general $M$-estimators.

We point out that it is up to the practitioner to determine the specific model and parameter estimation method, which are the starting point to carry out any subsequent 
CVaR estimation and inference. This parallels to what we usually do in time series forecasting based on some estimated model for the data. Our semiparametric CVaR estimator hinges on a preliminary $\sqrt{n}$-consistent estimator $\hat{\theta}$ of $\theta$, and our asymptotic confidence interval relies on the Bahadur representation of $\hat{\theta}-\theta$. In Section 4 below, we propose a sieve bootstrap approach, which can bypass such Bahadur representation.

\section{CVaR Process and Conditional Expected Shortfall}

In financial risk management, the portfolio manager may be interested in different percentiles (e.g., the top 1, 5, 10, 25-th percentiles) of the potential loss and draw some simultaneous inference. This type of information provides the basis for dynamically managing the portfolio to control the overall risk at different levels. This motivates us to study the CVaR process or equivalently the conditional quantile process $\{Q(\tau \mid x)\}_{\tau}$ on some quantile interval $\tau \in[\delta, 1-\delta]$ with some small $\delta>0$. Theorem 4 establishes a functional CLT version of Theorem 2 .

Theorem 4. Consider $[\delta, 1-\delta]$ with any small $\delta>0$. Suppose that Assumptions 1-2 and Assumptions 3-4 and $5^{*}$ in Section 8.1 hold. Then the functional CLT holds

$$
\{\sqrt{n}[\hat{Q}(\tau \mid x)-Q(\tau \mid x)]\}_{\tau \in[\delta, 1-\delta]} \Rightarrow\{Z(\tau)\}_{\tau \in[\delta, 1-\delta]} .
$$

Here $\{Z(\tau)\}$ is a centered Gaussian process with autocovariance (recall $W_{i}(\tau)$ in $\left.(17)\right)$ :

$$
\Gamma\left(\tau, \tau^{\prime}\right):=\operatorname{cov}\left\{Z(\tau), Z\left(\tau^{\prime}\right)\right\}=\dot{G}_{\varepsilon}\left(\theta, Q_{\varepsilon}(\tau), x\right) \dot{G}_{\varepsilon}\left(\theta, Q_{\varepsilon}\left(\tau^{\prime}\right), x\right) \operatorname{cov}\left\{W_{1}(\tau), W_{1}\left(\tau^{\prime}\right)\right\}
$$

Remark 2. For the quantile interval $[\delta, 1-\delta]$ in Theorem $4, \delta>0$ is assumed to be a given small number to avoid the boundary issue. We conjecture that, using more sophisticated arguments, it may be possible to extend the interval to $(0,1)$. The main technical issue is to establish the Bahadur representation (74) (see Section 8.3 in the proof section) uniformly for $\tau$ in some expanding interval $\left[\delta_{n}, 1-\delta_{n}\right]$ with $\delta_{n} \rightarrow 0$. For example, Portnoy and Koenker (1989) obtained such results for linear models. To avoid technical difficulties, we shall not pursue this direction. Also, in practice the most popular VaR levels we normally consider are $1-\tau=1 \%$ or $5 \%$, substantially below which any estimator may become unstable due to scarce observations in the extreme tail. 
As in Section 2.3, we can estimate the covariance function $\Gamma\left(\tau, \tau^{\prime}\right)$ in $(28)$ by

$$
\hat{\Gamma}\left(\tau, \tau^{\prime}\right)=\dot{G}_{\varepsilon}\left(\hat{\theta}, \hat{Q}_{\varepsilon}(\tau), x\right) \dot{G}_{\varepsilon}\left(\hat{\theta}, \hat{Q}_{\varepsilon}\left(\tau^{\prime}\right), x\right) \frac{1}{n-1} \sum_{i=1}^{n}\left[\widehat{W}_{i}(\tau)-\bar{W}(\tau)\right]\left[\widehat{W}_{i}\left(\tau^{\prime}\right)-\bar{W}\left(\tau^{\prime}\right)\right]
$$

where $\widehat{W}_{i}(\tau)$ and $\bar{W}(\tau)$ are defined in (19). Similar to Theorem 3, the uniform consistency of $\hat{\Gamma}\left(\tau, \tau^{\prime}\right)$ can be established along the similar line of argument. We omit the details.

The functional CLT in Theorem 4 provides a theoretical basis for simultaneous inference of CVaR at multiple levels. Here we consider an application to the expected shortfall (hereafter, ES), another risk measure in risk management. Recall that $\operatorname{CVaR}(1-\tau \mid x)$ is the $(1-\tau)$ worst scenario portfolio loss, given the covariates $\mathbf{X}_{i}=x$. At level $\gamma$, conditioning on $\mathbf{X}_{i}=x$, the conditional ES (hereafter, CES) is

$$
\operatorname{CES}(\gamma \mid x)=\frac{1}{\gamma} \int_{0}^{\gamma} \operatorname{CVaR}(\tau \mid x) d \tau=\frac{1}{\gamma} \int_{1-\gamma}^{1} \operatorname{CVaR}(1-\tau \mid x) d \tau .
$$

$\operatorname{CES}(\gamma \mid x)$ can be interpreted as the average conditional loss given that $\mathbf{X}_{i}=x$ and that the loss is at or even more extreme than the $100 \gamma$-th percentile worst scenario. Some recent nonparametric approaches include Scaillet (2004) and Chen (2008) for nonparametric ES estimation and Scaillet (2005) and Cai and Wang (2008) for nonparametric CES estimation.

In (30), the CVaR at all confidence levels are equally weighted, however practitioners may favor some confidence levels more than other levels. Let $\mathcal{T}$ be an interval of confidence level and $w(\cdot)$ a square-integrable weight function (depending on the practitioner's preference). We generalize (30) to the the weighted version on $\mathcal{T}$ :

$$
\operatorname{CES}(\mathcal{T} \mid x)=\int_{\mathcal{T}} w(1-\tau) \operatorname{CVaR}(1-\tau \mid x) d \tau, \quad \text { where } \quad \int_{\mathcal{T}} w(1-\tau) d \tau=1
$$

Clearly, (30) is a special case of $(31)$ with $w(\cdot) \equiv 1 / \gamma$ and $\mathcal{T}=[1-\gamma, 1]$. Since $\operatorname{CVaR}(1-$ $\tau \mid x)=Q(\tau \mid x)$, plugging in the estimator $\hat{Q}(\tau \mid x)$ in $(13)$, we estimate $\operatorname{CES}(\mathcal{T} \mid x)$ by

$$
\widehat{\mathrm{CES}}(\mathcal{T} \mid x)=\int_{\mathcal{T}} w(1-\tau) \hat{Q}(\tau \mid x) d \tau
$$

Remark 3. All results stated here also hold when $\mathcal{T}$ is a discrete set of confidence levels. In this case, we simply replace the integrals in (31)-(32) by summation over $\mathcal{T}$.

By Theorem 4 and the continuous mapping theorem, we can immediately obtain 
Theorem 5. Assume the same conditions in Theorem 4. For any interval $\mathcal{T} \subset[\delta, 1-\delta]$,

$$
\sqrt{n}[\widehat{\mathrm{CES}}(\mathcal{T} \mid x)-\operatorname{CES}(\mathcal{T} \mid x)] \Rightarrow \int_{\mathcal{T}} w(1-\tau) Z(\tau) d \tau
$$

where $\{Z(\tau)\}$ is the Gaussian process in Theorem 4 .

It is easy to see that the limiting distribution in (33) is a centered normal distribution with variance

$$
\iint_{\mathcal{T} \times \mathcal{T}} w(1-\tau) w\left(1-\tau^{\prime}\right) \Gamma\left(\tau, \tau^{\prime}\right) d \tau d \tau^{\prime}
$$

Here $\Gamma\left(\tau, \tau^{\prime}\right)$ is the covariance function defined in (28). This variance can be estimated by plugging the estimator $\hat{\Gamma}\left(\tau, \tau^{\prime}\right)$ in $(29)$.

\section{User-friendly Sieve Bootstrap Inference}

As discussed in Section 2.4, in order to implement the variance estimator $\hat{\Gamma}(\tau)$ in $(19)$ for the confidence interval (21), we need to know the Bahadur representation (15); the same requirement is also needed for the covariance estimator $\hat{\Gamma}\left(\tau, \tau^{\prime}\right)$ in (29) [however, the CVaR estimator $\hat{Q}(\tau \mid x)$ in (13) does not require this]. It may be non-trivial for a non-expert practitioner to derive a Bahadur representation for their specific model and parameter estimation. In this section we provide a user-friendly bootstrap approach.

For time series, two popular bootstrap methods are the block bootstrap (Lahiri, 2003) and the sieve bootstrap (Bühlmann, 1997). The block bootstrap requires the challenging issue of block length selection. For a given time series model subject to unknown parameters, the sieve bootstrap creates bootstrap samples by recursively using the model with estimated parameters and resampled residuals, and thus the bootstrap data can preserve the dependence structure of the original data. Here we adopt the sieve bootstrap.

Assume that the covariates $\mathbf{X}_{i}=\left(Y_{i-1}, \ldots, Y_{i-q}, \mathbf{U}_{i}\right)$ consist of both lagged $Y$ 's and some other covariates $\mathbf{U}_{i}$. We propose the following sieve bootstrap procedure:

(i) Use some parameter estimation method to obtain the estimate $\hat{\theta}$ and then compute $\left\{\hat{\varepsilon}_{i}\right\}[$ see $(11)]$ and $\hat{Q}(\tau \mid x)[$ see $(13)]$ based on the original data. 
(ii) Obtain the bootstrap samples $\left\{\left(\mathbf{X}_{i}^{*}, Y_{i}^{*}\right)\right\}$ recursively (with same initial values as $\mathbf{X}_{i}$ )

$$
Y_{i}^{*}=G\left(\hat{\theta}, \varepsilon_{i}^{*}, \mathbf{X}_{i}^{*}\right) \quad \text { with } \quad \mathbf{X}_{i}^{*}=\left(Y_{i-1}^{*}, \ldots, Y_{i-q}^{*}, \mathbf{U}_{i}\right)
$$

where $\left\{\varepsilon_{i}^{*}\right\}$ are i.i.d. random samples (with replacement) from $\left\{\hat{\varepsilon}_{i}\right\}$. Use the bootstrap data $\left\{\left(\mathbf{X}_{i}^{*}, Y_{i}^{*}\right)\right\}$ and the same parameter estimation method in step (i) to obtain new parameter estimate $\hat{\theta}^{*}$ and new conditional quantile estimate $\hat{Q}^{*}(\tau \mid x)$.

(iii) Repeat (ii) to obtain a large number $\left(M\right.$, say) of realizations of $\hat{Q}^{*}(\tau \mid x)$, denoted by $\hat{Q}^{*(1)}(\tau \mid x), \ldots, \hat{Q}^{*(M)}(\tau \mid x)$.

We make one important comment about the bootstrap procedure in prediction setting. Suppose we wish to construct bootstrap interval for the predictive quantile $Q\left(\tau \mid\left\{Y_{i}\right\}_{i \leq n}\right)$ for $Y_{n+1}$ based on data $\left\{Y_{i}\right\}_{i=1}^{n}$ from the GARCH model $Y_{i}=\sigma_{i} \varepsilon_{i}, \sigma_{i}^{2}=\omega+\alpha Y_{i-1}^{2}+\beta \sigma_{i-1}^{2}$. In step (i) above, we fit GARCH model to obtain estimates $(\hat{\omega}, \hat{\alpha}, \hat{\beta}), \hat{\sigma}_{i}$, and $\hat{\varepsilon}_{i}=Y_{i} / \hat{\sigma}_{i}$. In step (ii), first we generate bootstrap samples $\left\{Y_{i}^{*}\right\}_{i=1}^{n}$ from $Y_{i}^{*}=\sigma_{i}^{*} \varepsilon_{i}^{*}, \sigma_{i}^{* 2}=\hat{\omega}+\hat{\alpha} Y_{i-1}^{* 2}+$ $\hat{\beta} \sigma_{i-1}^{* 2}$, then fit GARCH model to $\left\{Y_{i}^{*}\right\}_{i=1}^{n}$ to obtain estimates $\hat{\sigma}_{i}^{*}$ and $\hat{\varepsilon}_{i}^{*}=Y_{i}^{*} / \hat{\sigma}_{i}^{*}$, and finally compute $\hat{Q}^{*}\left(\tau \mid\left\{Y_{i}\right\}_{i \leq n}\right)$ as $\hat{\sigma}_{n+1}$ multiplied by the sample $\tau$-th quantile of $\left\{\hat{\varepsilon}_{i}^{*}\right\}_{i=1}^{n}$. It is important to use $\hat{\sigma}_{n+1}$ instead of $\hat{\sigma}_{n+1}^{*}$. This is because $Q\left(\tau \mid\left\{Y_{i}\right\}_{i \leq n}\right)$ is the $\tau$-th quantile of $Y_{n+1}$ given fixed covariates $\left\{Y_{i}\right\}_{i \leq n}$ and $\hat{\sigma}_{n+1}$ reflects such fixed covariates. By contrast, using $\hat{\sigma}_{n+1}^{*}$ would mean that we are estimating $Q\left(\tau \mid\left\{Y_{i}^{*}\right\}_{i \leq n}\right)$ instead of $Q\left(\tau \mid\left\{Y_{i}\right\}_{i \leq n}\right)$.

We discuss some bootstrap inference below.

\section{Bootstrap confidence interval for CVaR}

For the realizations $\hat{Q}^{*(1)}(\tau \mid x), \ldots, \hat{Q}^{*(M)}(\tau \mid x)$ in step (iii) above, denote by $q_{1-\alpha}^{*}$ the $(1-\alpha)$ sample quantile of $\sqrt{n}\left|\hat{Q}^{*(1)}(\tau \mid x)-\hat{Q}(\tau \mid x)\right|, \ldots, \sqrt{n}\left|\hat{Q}^{*(M)}(\tau \mid x)-\hat{Q}(\tau \mid x)\right|$. Then the $(1-\alpha)$ bootstrap confidence interval for $Q(\tau \mid x)$ is

$$
\hat{Q}(\tau \mid x) \pm q_{1-\alpha}^{*} / \sqrt{n}
$$

\section{Bootstrap confidence interval for CES}

Plugging the realizations $\hat{Q}^{*(1)}(\tau \mid x), \ldots, \hat{Q}^{*(M)}(\tau \mid x)$ into (32) to obtain the bootstrapdata-based CES estimates $\widehat{\mathrm{CES}}^{*(1)}(\mathcal{T} \mid x), \ldots, \widehat{\mathrm{CES}}^{*(M)}(\mathcal{T} \mid x)$. Denote by $r_{1-\alpha}$ the $(1-\alpha)$

sample quantile of $\sqrt{n}\left|\widehat{\operatorname{CES}}^{*(1)}(\mathcal{T} \mid x)-\widehat{\mathrm{CES}}(\mathcal{T} \mid x)\right|, \ldots, \sqrt{n}\left|\widehat{\mathrm{CES}}^{*(M)}(\mathcal{T} \mid x)-\widehat{\mathrm{CES}}(\mathcal{T} \mid x)\right|$. Then the $(1-\alpha)$ bootstrap confidence interval for $\operatorname{CES}(\mathcal{T} \mid x)$ is

$$
\widehat{\mathrm{CES}}(\mathcal{T} \mid x) \pm r_{1-\alpha} / \sqrt{n}
$$


The easy-to-implement bootstrap confidence intervals (36)-(37) require only some pa-

rameter estimation method for $\hat{\theta}$, and thus practitioners can construct CVaR and CES confidence intervals using our proposed semiparametric CVaR and CES estimator along with their favorite parameter estimation methods. Since the bootstrap model (35) inherits the same structure of the original model (4), the bootstrap data can closely mimic the dependence structure of the original data. Our simulation study in Section 6 suggests that the bootstrap confidence intervals have better finite sample performance than the asymptotic confidence intervals based on estimated limiting variances.

\section{Semiparametric conditional distribution estimation}

In this section we adopt the semiparametric approach in Section 2 to study conditional distribution estimation. Denote by $F(y \mid x)=\mathbb{P}\left\{Y_{i} \leq y \mid \mathbf{X}_{i}=x\right\}$ the conditional distribution function of $Y_{i}$ given $\mathbf{X}_{i}=x$. The conditional distribution can fully characterize the distributional dependence of the loss $Y_{i}$ on the covariates $\mathbf{X}_{i}$.

To estimate $F(y \mid x)$, the usual nonparametric kernel regression approach is

$$
\tilde{F}(y \mid x)=\frac{\sum_{i=1}^{n} \mathbf{1}_{Y_{i} \leq y} K\left\{\left(\mathbf{X}_{i}-x\right) / b_{n}\right\}}{\sum_{i=1}^{n} K\left\{\left(\mathbf{X}_{i}-x\right) / b_{n}\right\}},
$$

where $K(\cdot)$ and $b_{n}$ are the kernel and bandwidth as in Section 2.1; see Chapter 6 in Li and Racine (2007). This nonparametric conditional distribution estimate has the same drawback as the nonparametric conditional quantile estimation in Section 2.1.

We can easily adapt our method in Section 2.2 to construct a $\sqrt{n}$-consistent estimate of $F(y \mid x)$. Under Assumption 1 and by the independence between $\varepsilon_{i}$ and $\mathbf{X}_{i}$,

$$
\mathbb{P}\left\{Y_{i} \leq y \mid \mathbf{X}_{i}=x\right\}=\mathbb{P}\left\{G\left(\theta, \varepsilon_{i}, x\right) \leq y\right\}=\mathbb{P}\left\{\varepsilon_{i} \leq H(\theta, y, x)\right\}=F_{\varepsilon}\{H(\theta, y, x)\}
$$

Therefore we propose the following semiparametric estimate of $F(y \mid x)$ :

$$
\hat{F}(y \mid x)=\hat{F}_{\varepsilon}\{H(\hat{\theta}, y, x)\}
$$

where $\hat{\theta}$ is a consistent estimate of $\theta$, and $\hat{F}_{\varepsilon}(z)$ [defined in (12)] is the sample empirical distribution of the generalized residuals $\left\{\hat{\varepsilon}_{i}\right\}$. Theorem 6 below presents a functional CLT, which implies the pointwise CLT. 
Theorem 6. Suppose that the same conditions in Theorem 4 hold. Let $\mathcal{Y}=\left[\mathcal{Y}_{1}, \mathcal{Y}_{2}\right]$ be any bounded interval. Then the functional CLT holds

$$
\{\sqrt{n}[\hat{F}(y \mid x)-F(y \mid x)]\}_{y \in \mathcal{Y}} \Rightarrow\{S(y)\}_{y \in \mathcal{Y}}
$$

where $\{S(y)\}_{y \in \mathcal{Y}}$ is a centered Gaussian process with autocovariance

$$
\Sigma\left(y, y^{\prime}\right):=\operatorname{cov}\left\{S(y), S\left(y^{\prime}\right)\right\}=\operatorname{cov}\left\{V_{1}(y), V_{1}\left(y^{\prime}\right)\right\}
$$

and

$$
\begin{aligned}
V_{i}(y)= & {\left[\mathbf{1}_{\varepsilon_{i} \leq H(\theta, y, x)}-\mathbb{E}\left(\mathbf{1}_{\varepsilon_{i} \leq H(\theta, y, x)}\right]\right.} \\
& +f_{\varepsilon}(H(\theta, y, x))\left\{\mathbb{E}\left[\frac{\dot{G}_{\theta}\left(\theta, H(\theta, y, x), \mathbf{X}_{0}\right)}{\dot{G}_{\varepsilon}\left(\theta, H(\theta, y, x), \mathbf{X}_{0}\right)}\right]+\dot{H}(\theta, y, x)\right\}^{T} D\left(\theta, \varepsilon_{i}, \mathbf{X}_{i}\right) .
\end{aligned}
$$

In (43), the first component is from the empirical distribution of the true innovations $\left\{\varepsilon_{i}\right\}$, and the second component is due to the estimation error of $\hat{\theta}$. Compared to the nonparametric kernel smoothing estimator in (38), the proposed semiparametric estimator in (40) is easy to implement and attains $\sqrt{n}$ parametric convergence rate.

Similar to the estimation of $W_{i}(\tau)$ in Section 2.2, we can estimate $V_{i}(y)$ by

$$
\begin{aligned}
\widehat{V}_{i}(y)= & {\left[\mathbf{1}_{\varepsilon_{i} \leq H(\hat{\theta}, y, x)}-\hat{F}_{\varepsilon}(H(\hat{\theta}, y, x))\right] } \\
& +\hat{f}_{\varepsilon}(H(\hat{\theta}, y, x))\left\{\frac{1}{n} \sum_{i=1}^{n} \frac{\dot{G}_{\theta}\left(\hat{\theta}, H(\hat{\theta}, y, x), \mathbf{X}_{i}\right)}{\dot{G}_{\varepsilon}\left(\hat{\theta}, H(\hat{\theta}, y, x), \mathbf{X}_{i}\right)}+\dot{H}(\hat{\theta}, y, x)\right\}^{T} D\left(\hat{\theta}, \hat{\varepsilon}_{i}, \mathbf{X}_{i}\right) .
\end{aligned}
$$

Then the covariance in (42) can be estimated by the sample covariance

$$
\hat{\Sigma}\left(y, y^{\prime}\right)=\frac{1}{n-1} \sum_{i=1}^{n}\left[\widehat{V}_{i}(y)-\bar{V}(y)\right]\left[\widehat{V}_{i}\left(y^{\prime}\right)-\bar{V}\left(y^{\prime}\right)\right], \quad \bar{V}(y)=\frac{1}{n} \sum_{i=1}^{n} \widehat{V}_{i}(y) .
$$

Similar to Theorem 3, we can establish the consistency of $\hat{\Sigma}\left(y, y^{\prime}\right)$. Also, the sieve bootstrap procedure in Section 4 can be applied here to avoid the issue of Bahadur representation.

\section{Monte Carlo Studies}

\subsection{MISE comparison with nonparametric method}

For the nonparametric estimator $\tilde{Q}(\tau \mid x)$ in $(3)$, let $K(\cdot)$ be the $p$-variate standard normal density. Using 300 realizations $\tilde{Q}^{(1)}(\tau \mid x), \ldots, \tilde{Q}^{(300)}(\tau \mid x)$ of $\tilde{Q}(\tau \mid x)$, we measure the 
performance of $\tilde{Q}(\tau \mid x)$ by the empirical mean integrated squared error (MISE) on a set $\mathcal{X}$ :

$$
\operatorname{MISE}\left\{\tilde{Q}(\tau \mid \cdot) ; b_{n}\right\}=\frac{1}{300} \sum_{\ell=1}^{300} \int_{\mathcal{X}}\left[\tilde{Q}^{(\ell)}(\tau \mid x)-Q(\tau \mid x)\right]^{2} d x .
$$

For the semiparametric estimator $\hat{Q}(\tau \mid x)$ in (13), its MISE is defined similarly. We further define the relative MISE (RMISE) of $\hat{Q}(\tau \mid x)$, relative to the nonparametric estimator $\tilde{Q}(\tau \mid x)$ under the best-case scenario, as

$$
\operatorname{RMISE}=\frac{\min _{b_{n}} \operatorname{MISE}\left\{\tilde{Q}(\tau \mid \cdot) ; b_{n}\right\}}{\operatorname{MISE}\{\hat{Q}(\tau \mid \cdot)\}} .
$$

Here, we use the theoretical optimal bandwidth to minimize $\operatorname{MISE}\left\{\tilde{Q}(\tau \mid \cdot) ; b_{n}\right\}$. The comparison will favor the nonparametric method as the choice of bandwidth for $\tilde{Q}(\tau \mid x)$ is done under the best-case scenario, which is generally unavailable in practice. A value of RMISE $\geq 1$ indicates better MISE performance of the proposed method.

We consider the following four increasingly more complicated models:

Model 1: $Y_{i}=\theta_{0}+\theta_{1} Y_{i-1}+\sigma \varepsilon_{i}, \quad\left(\theta_{0}, \theta_{1}, \sigma\right)=(0.3,0.4,0.5)$;

Model 2: $\quad Y_{i}=\theta_{0}+\theta_{1} Y_{i-1}+\theta_{2} Y_{i-2}+\sigma_{i} \varepsilon_{i}, \quad\left(\theta_{0}, \theta_{1}, \theta_{2}, \sigma\right)=(0.3,0.4,-0.5,0.5)$;

Model 3: $Y_{i}=\theta_{0}+\theta_{1} Y_{i-1}+\varepsilon_{i} \sqrt{\theta_{2}^{2}+\theta_{3}^{2} Y_{i-1}^{2}}, \quad\left(\theta_{0}, \theta_{1}, \theta_{2}, \theta_{3}\right)=(0.3,0.4,0.3,0.5)$;

Model 4: $\quad Y_{i}=\theta_{0}+\theta_{1} Y_{i-1}+\gamma_{1} U_{i}+\varepsilon_{i} \sqrt{\theta_{2}^{2}+\theta_{3}^{2} Y_{i-1}^{2}+\gamma_{2}^{2} U_{i}^{2}}, \quad U_{i}:$ uniform [0,1], with $\left(\theta_{0}, \theta_{1}, \theta_{2}, \theta_{3}, \gamma_{1}, \gamma_{2}\right)=(0.3,0.4,0.3,0.5,-0.4,0.3)$.

Model 1 and 2 are simple AR(1) and AR(2) models, Model 3 is an AR(1)-ARCH(1) model, and Model 4 is an $\mathrm{AR}(1)-\mathrm{ARCH}(1)$ with exogenous variable $U_{i}$. The AR-ARCH model with exogenous input allows us to model how stock returns depend on the past returns as well as other external variables. The noise $\varepsilon_{i}$ is from two distributions: (i) standard normal $N(0,1)$, and (ii) $t_{3} / \sqrt{3}$ (Student- $t$ distribution with 3 degrees of freedom with the normalizer $\sqrt{3}$ making the variance one). In all settings we use sample size $n=200$.

For Model 1 and 3, we estimate the conditional $\tau$-th quantile of $Y_{i}$ given $Y_{i-1}=x$, and we take $\mathcal{X}$ in (45) to be the range of 2.5-th and 97.5-th percentiles of $\left\{Y_{i-1}\right\}$; for Model 2 (resp. Model 4), we estimate the conditional $\tau$-th quantile of $Y_{i}$ given the bivariate $\mathbf{X}_{i}:=\left(Y_{i-1}, Y_{i-2}\right)=\left(x_{1}, x_{2}\right)\left(\right.$ resp. $\mathbf{X}_{i}=\left(Y_{i-1}, U_{i}\right)$ for Model 4), and we take $\mathcal{X}$ in $(45)$ to 
be $\mathcal{X}_{1} \times \mathcal{X}_{2}$, where $\mathcal{X}_{1}$ and $\mathcal{X}_{2}$ are, respectively, the range of 2.5-th and 97.5 -th percentiles of each of the two coordinates of $\mathbf{X}_{i}$. The integral in (45) is approximated by 20 evenly spaced grid points in the univariate case (Model 1 and 3) or $10 \times 10$ evenly spaced grid points in the bivariate case (Model 2 and 4). To implement the semiparametric method, we use (23) to estimate the unknown parameters. The procedure is repeated for 13 different quantiles $\tau=1 \%, 5 \%, 10 \%, \ldots, 90 \%, 95 \%, 99 \%$.

\section{Insert Table 1 here.}

Table 1 summarizes the RMISE [see (46)]. For almost all cases considered, a substantial MISE improvement can be achieved by using the semiparametric CVaR estimator. The MISE improvement is more significant for the extreme quantiles $\tau=90 \%, 95 \%, 99 \%$, which correspond to the most widely used confidence levels $1-\tau=10 \%, 5 \%, 1 \%$ in the $\mathrm{VaR}$ literature. For the middle-range quantiles $\tau=20 \%, \ldots, 80 \%$, the semiparametric estimator significantly outperforms the nonparametric estimator for $N(0,1)$ noise, whereas the two methods have comparable performance for Student- $t$ noise. However, we emphasize that the MISE comparison is done between the proposed method and the nonparametric method under the best-case scenario. In practice, the optimal bandwidth is generally unknown, therefore the proposed estimator can deliver relatively even better performance.

\subsection{Coverage rate evaluation}

To evaluate VaR estimator, another criterion is the empirical coverage rate, i.e., the empirical proportion, denoted by $\hat{\tau}$, of realizations such that $Y_{i} \leq \hat{Q}\left(\tau \mid \mathbf{X}_{i}\right)$. Specifically, our empirical coverage rate is calculated as follows:

(i) For each realization $\left\{\left(\mathbf{X}_{i}, Y_{i}\right)\right\}_{i=1}^{n}$, we use observations $\left\{\left(\mathbf{X}_{i}, Y_{i}\right)\right\}_{i=1}^{n-1}$ up to time $n-1$ to fit the model and estimate the predicted $\tau$-th quantile $\hat{Q}\left(\tau \mid \mathbf{X}_{n}\right)$ for $Y_{n}$. We then check whether $Y_{n} \leq \hat{Q}\left(\tau \mid \mathbf{X}_{n}\right)$.

(ii) Repeat (i) 1000 times and compute the empirical coverage rate $\hat{\tau}$ as the proportion of realizations such that $Y_{n} \leq \hat{Q}\left(\tau \mid \mathbf{X}_{n}\right)$.

By definition, the closer $\hat{\tau}$ to $\tau$, the better performance of $\hat{Q}\left(\tau \mid \mathbf{X}_{i}\right)$. 
The four models in Section 6.1 are ARCH-type models, and in this section we examine some GARCH-type models of the form (8). We consider three GARCH models:

Model 5: $\quad Y_{i}=\sigma_{i} \varepsilon_{i}, \quad \sigma_{i}^{2}=\omega+\alpha Y_{i-1}^{2}+\beta \sigma_{i-1}^{2}$;

Model 6: $\quad Y_{i}=\sigma_{i} \varepsilon_{i}, \quad \log \left(\sigma_{i}^{2}\right)=\omega+\alpha \frac{Y_{i-1}}{\sigma_{i-1}}+\beta \log \left(\sigma_{i-1}^{2}\right)+\gamma\left[\frac{\left|Y_{i-1}\right|}{\sigma_{i-1}}-\mathbb{E}\left(\frac{\left|Y_{i-1}\right|}{\sigma_{i-1}}\right)\right]$;

Model 7: $\quad Y_{i}=\sigma_{i} \varepsilon_{i}, \quad \sigma_{i}^{2}=\omega+\alpha Y_{i-1}^{2}+\beta \sigma_{i-1}^{2}+\gamma Y_{i-1}^{2} \mathbf{1}_{Y_{i-1}<0}$.

Model 5 is the standard GARCH model (Bollerslev, 1986), Model 6 is the EGARCH model (Nelson, 1991), and Model 7 is the GJR-GARCH model (Glosten, Jagannathan and Runkle, 1993). In Model 5, $(\omega, \alpha, \beta)=(0.1,0.3,0.5)$; In Model $6,(\omega, \alpha, \beta, \gamma)=$ $(-3,-0.4,0.5,0.3)$; in Model $7,(\omega, \alpha, \beta, \gamma)=(0.1,0.3,0.5,0.2)$. As in Models 1-4, we consider two distributions, $N(0,1)$ and $t_{3} / \sqrt{3}$, for the noise $\varepsilon_{i}$.

As discussed in Example 3, GARCH models are non-Markovian and it is infeasible to use the nonparametric approach. Thus, we only evaluate the coverage rate for the proposed semiparametric method. When using the $\mathrm{R}$ package rugarch (Ghalanos, 2014) to do parameter estimation, we always specify the noise distribution as normal, regardless of the actual noise distribution $\left[N(0,1)\right.$ or $\left.t_{3} / \sqrt{3}\right]$. That is, for $t_{3} / \sqrt{3}$-distributed noises, the parameter estimation is done under mis-specification of the noise distribution.

Based on 1000 realizations for each setting, Table 2 summarizes the empirical coverage rate at two sample sizes $n=200$ and $n=500$. Overall, the empirical coverage rate is close to the nominal level, and the larger sample size $n=500$ leads to better performance.

\section{Insert Table 2 here.}

\subsection{Comparison with parametric-distribution based competitors}

Many existing VaR methods use parametric distribution for the noise $\varepsilon_{i}$ in the model. For example, the EWMA in RiskMetrics of J.P. Morgan (1994) uses standard Normal distribution. Different approaches have been proposed to model the observed heavy-tail and asymmetric returns, including the Student- $t$ distribution, Laplace-distribution based robust-EWMA in Guermat and Harris (2001), and the asymmetric-Laplace distribution based skewed-EWMA in Gerlach, Lu and Huang (2013). Both the proposed semiparametric approach and the aforementioned existing methods require a parametric specification 
on the model structure, but our semiparametric approach does not impose any parametricdistribution on the noises.

To appreciate the advantage of the nonparametric distribution approach (i.e., using the sample quantile in (12)), we compare it with three parametric-distribution based methods: (i) Normal distribution; (ii) Student- $t$ distribution; and (iii)Asymmetric-Laplacedistribution (Gerlach, Lu and Huang, 2013), which includes the Laplace-distribution (Guermat and Harris, 2001) as a special case. The parameters in these parametric methods are estimated via the maximum likelihood method.

We compare the performance of these methods in estimating the $\tau$-th quantile, denoted by $Q_{\varepsilon}(\tau)$, of the noise distribution when the noise $\varepsilon_{i}$ comes from five distributions: $N(0,1)$, $t_{3} / \sqrt{3}$, Laplace $/ \sqrt{2}$ (the scaled Laplace with the factor $1 / \sqrt{2}$ making the variance one), Normal mixture $0.5 N(0,0.5)+0.5 N(0,1.5)$, and standard exponential minus 1 . All five distributions have variance one. In all settings we use sample size $n=1000$.

For the nonparametric sample quantile $\hat{Q}_{\varepsilon}(\tau)$ in (12), as in (46), we define the relative mean squared error (RMSE) of $\hat{Q}_{\varepsilon}(\tau)$ relative to a parametric method as

$$
\operatorname{RMSE}=\frac{\operatorname{MSE}\{\text { parametric method }\}}{\operatorname{MSE}\left\{\hat{Q}_{\varepsilon}(\tau)\right\}},
$$

where the empirical mean squared error (MSE) is calculated based on 1000 realizations. RMSE $\geq 1$ indicates better MSE performance of the nonparametric distribution method.

Table 3 summarizes the RMSE results for different $\tau$. When the noise distribution is correctly specified, parametric-distribution methods work well, which is not surprising since we use the maximum likelihood method to estimate the parameters. However, for misspecified noise distributions, parametric-distribution methods may suffer from seriously poor performance. By contrast, the nonparametric distribution method yields reasonable performance in all cases. For the practically most interesting case $\tau=95 \%$ or $1-\tau=5 \%$, the correctly specified parametric method can reduce the MSE of the nonparametric distribution method by about 50\%; however, in the presence of mis-specification, parametric methods can have MSE a few times larger than the nonparametric method. In practice, the noise distribution is usually unknown, thus we recommend the more robust nonparametric method to avoid potential mis-specification on the noise distribution.

\section{Insert Table 3 here.}




\subsection{Performance under model mis-specification}

The proposed semiparametric approach requires a parametrization on the model structure, and below we examine its performance under model mis-specification.

We consider the following model mis-specification:

true model: GJR-GARCH Model 7 with $(\omega, \alpha, \beta)=(0.1,0.3,0.5)$ and different $\gamma$; mis-specified model: standard GARCH Model 5.

The parameter $\gamma$ in GJR-GARCH model measures the deviation between the true and mis-specified model. If $\gamma=0$, then GJR-GARCH reduces to the standard GARCH model. Under this model mis-specification, we examine RMSE [see (47)] of the semiparametric method to the three parametric methods in Section 6.3 for predicting $\operatorname{CVaR}\left(1-\tau \mid Y_{j}, j \leq\right.$ $n-1)$ of $Y_{n}$, under different combinations of $\gamma, \tau$, and the five noise distributions in Section 6.3. Since the results for other settings are similar, Table 4 presents the results for $\gamma=0.2,0.4, \tau=60 \%, 70 \%, 80 \%, 90 \%, 95 \%, 99 \%$, and $n=1000$. Clearly, the proposed method delivers better overall performance than other methods. For example, for $\tau=95 \%$, the proposed method has either comparable or better performance than other methods.

\section{Insert Table 4 here.}

We also examined other model mis-specifications. For example, the true model is

$$
\text { AR-GARCH: } Y_{i}=\gamma Y_{i-1}+\sigma_{i} \varepsilon_{i} \quad \sigma_{i}^{2}=\omega+\alpha Y_{i-1}^{2}+\beta \sigma_{i-1}^{2}
$$

The mis-specified model is the standard GARCH. Again we found that the method outperforms other methods. To keep the length, we did not include the results here.

\subsection{Asymptotic versus bootstrap confidence intervals}

The finite sample performance of CVaR confidence intervals has not been examined in the literature; in this section we compare the performance of the asymptotic confidence interval and the bootstrap confidence interval for CVaR: the asymptotic confidence interval is based on the asymptotic normality in Theorem 2 with estimated limiting variance in Section 2.3, and the bootstrap confidence interval is constructed using the procedure in Section 4 with 
$M=1000$ bootstrap replications. The empirical coverage probability is the proportion of confidence intervals among 1000 realizations of $(1-\alpha)$ confidence intervals that cover the true CVaR $(1-\tau \mid x)$. Table 5 presents the results for the most typical setting $1-\tau=5 \%$ and $1-\alpha=90 \%, 95 \%, 99 \%$, at different values of $x$. Overall, the bootstrap confidence interval delivers much better performance and has empirical coverage probabilities close to the nominal levels. Given the easy implementation and superior empirical performance, we recommend the bootstrap confidence interval in practice.

\section{Insert Table 5 here.}

\section{An Empirical Application to S\&P 500 Index}

As an illustration, we consider S\&P 500 index daily loss defined as $Y_{i}=-\left[\log \left(S_{i}\right)-\right.$ $\left.\log \left(S_{i-1}\right)\right]$, where $S_{i}$ is the index at day $i$. Figure 1 is a plot of the loss series $\left\{Y_{i}\right\}$ over the ten-year time period January 2004-December, 2013. There are $n=2516$ observations. The plot clearly shows volatility clustering, so GARCH models are natural choices.

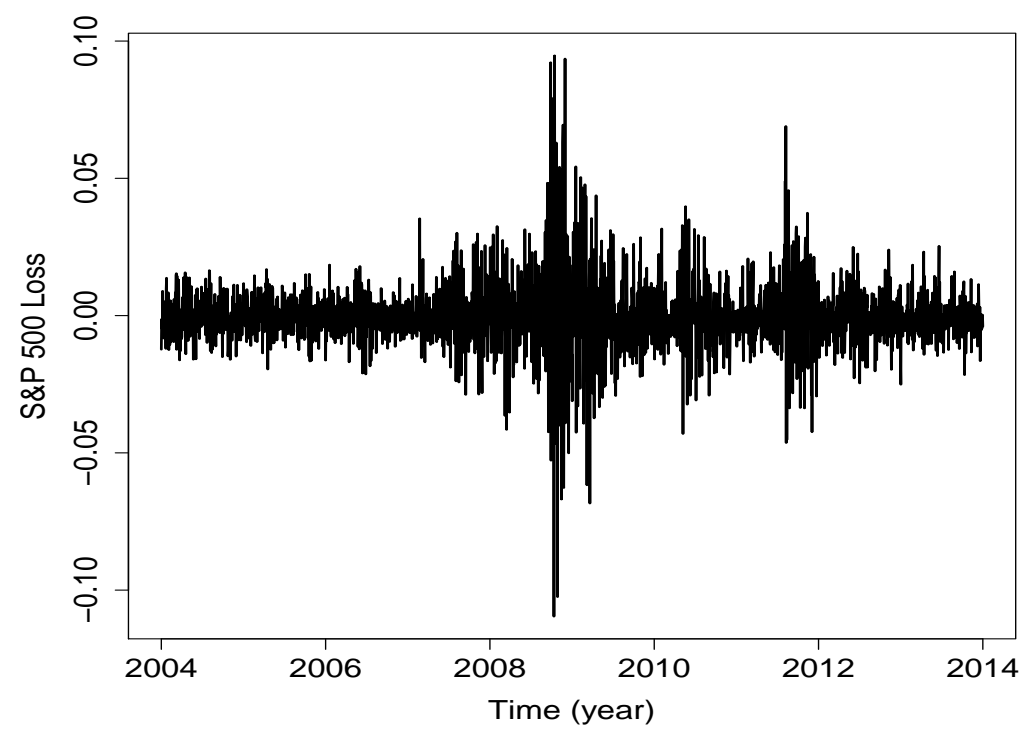

Figure 1: Time series plot of daily S\&P 500 index loss $\left\{Y_{i}\right\}_{i=1}^{n}$ (i.e., negative logarithm return) during the ten-year period January 2004-December, 2013. 


\subsection{Comparison under different GARCH models}

We consider sequential predictions of CVaR using three GARCH models: standard GARCH, EGARCH, and GJR-GARCH, as described in Model 5-7 in Section 6.2. For a given time $i$, based on the historical data $\mathbf{X}_{i}=\left\{Y_{j}\right\}_{j \leq i-1}$, we apply our semiparametric CVaR method to obtain the estimate $\widehat{\mathrm{CVaR}}\left(1-\tau \mid Y_{j}, j \leq i-1\right)$ for loss $Y_{i}$. Repeating the procedure for $n-(J-1) \leq i \leq n$, we obtain the sequentially predicted CVaR for the last $J=1000$ daily losses, which roughly corresponds to the daily losses during the last four years 2010-2013. Cai and Wang (2008) studied daily loss over the period 1998-2006 and nonparametrically estimated the CVaR curve for $Y_{i}$ conditioning on $Y_{i-1}=x$. In our setting, due to the non-Markovian structure of GARCH models, it is infeasible to use their nonparametric approach.

Using the three GARCH models, Figure 2 plots the corresponding sequential CVaR predictions at level $1-\tau=10 \%$ (top plot), $5 \%$ (middle plot), and 1\% (bottom plot). From Figure 2, at each level, the three CVaR curves based on standard GARCH, EGARCH, and GJR-GARCH exhibit quite similar pattern, indicating the robustness of our method to different choices of GARCH models. 
Level $10 \%$
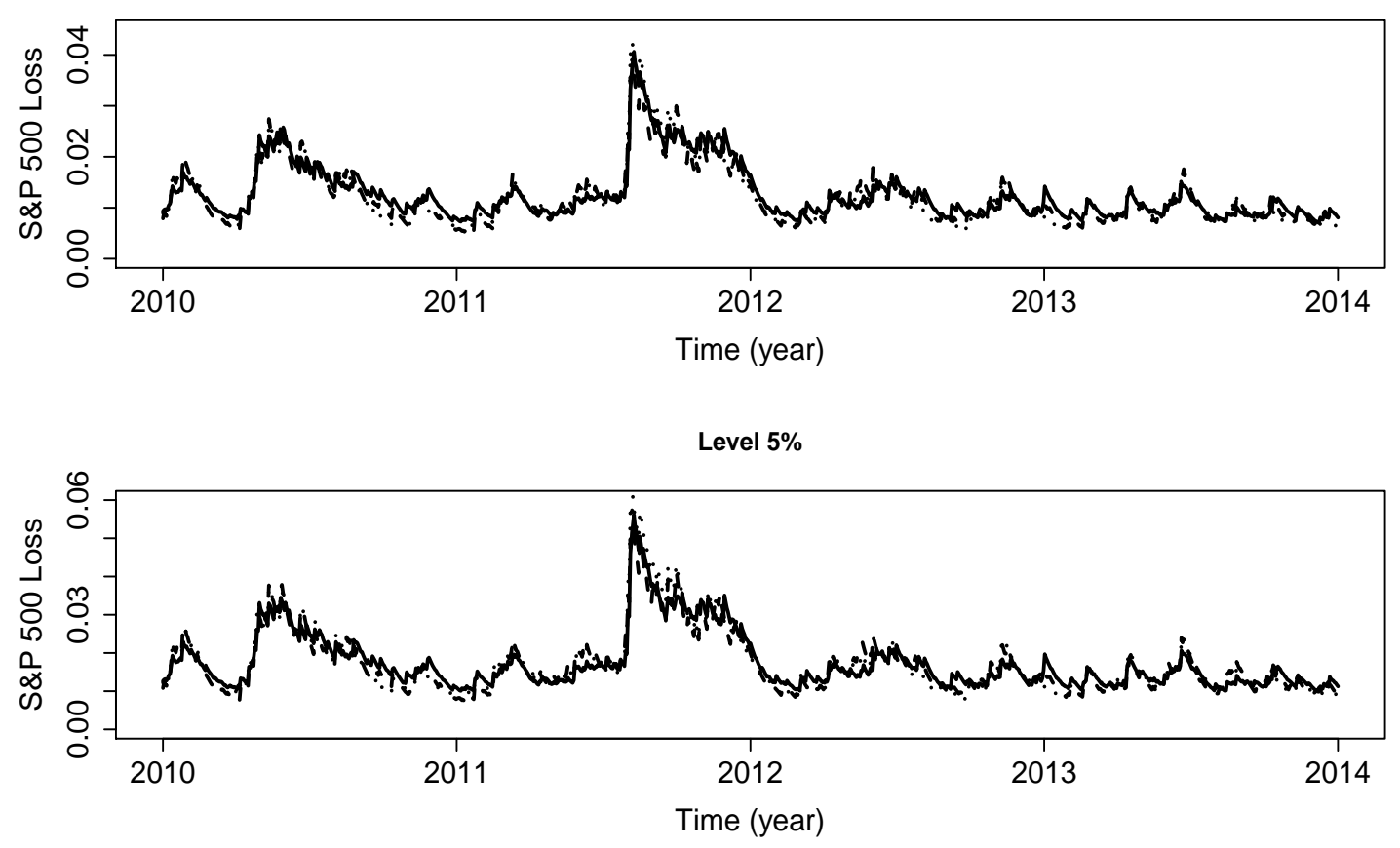

Level $1 \%$

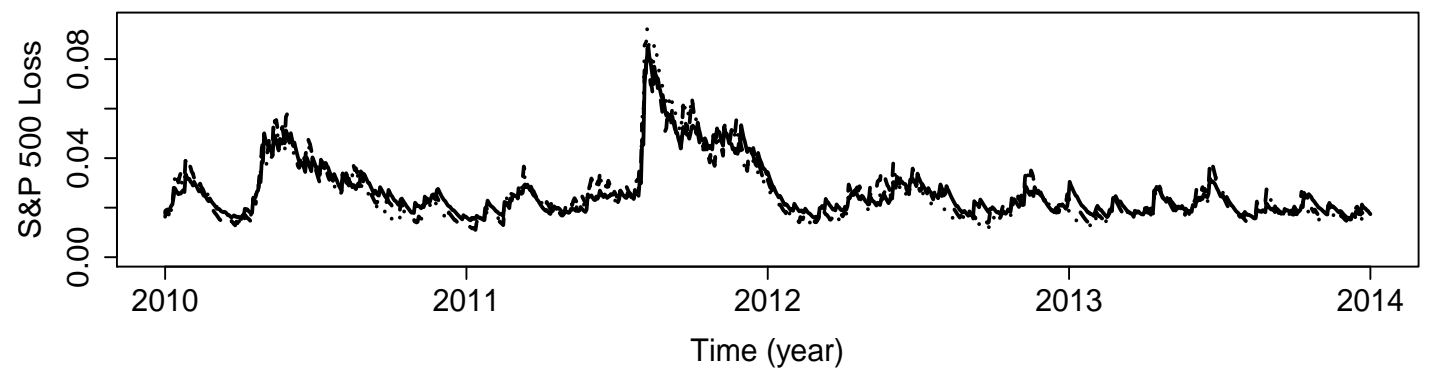

Figure 2: Sequentially predicted semiparametric CVaR for daily losses during 2010-2013, using standard GARCH (solid curve), EGARCH (dashed curve), and GJR-GARCH (dotted curve) models. Top, middle, and bottom plots correspond to level $1-\tau=10 \%, 5 \%, 1 \%$, respectively.

Despite the vast literature on $\mathrm{VaR} / \mathrm{CVaR}$ estimation, their confidence interval construction has been largely ignored. Using the bootstrap procedure in Section 4, Figure 3 presents the semiparametrically estimated CVaR at level 5\% and the corresponding pointwise $95 \%$ confidence interval. Due to the quite similar pattern of CVaR using different GARCH models, we only report the result for standard GARCH. 


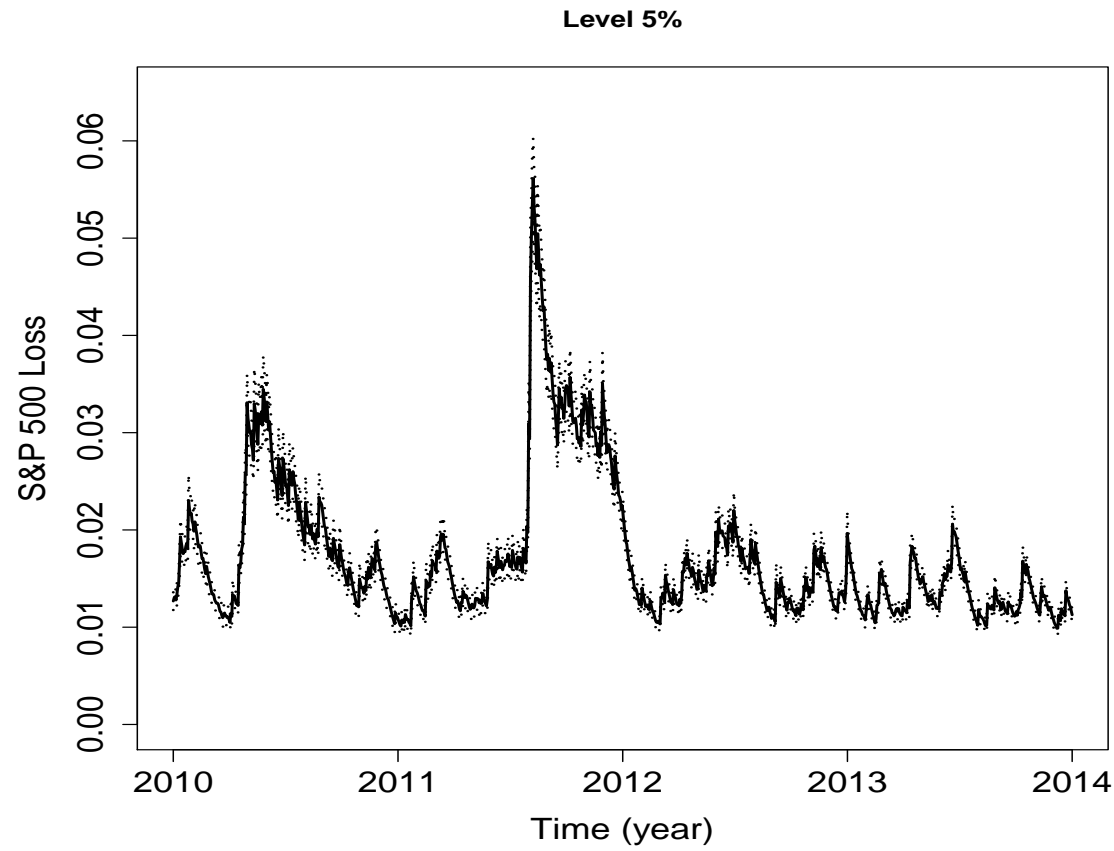

Figure 3: Sequentially predicted semiparametric CVaR (solid curve) at level $5 \%$ for daily losses during 2010-2013 using standard GARCH. The dotted curves are the pointwise bootstrap 95\% confidence interval.

\subsection{Comparison with some existing methods}

We compare our semiparametric CVaR predictions with three parametric-distribution based approaches: the EWMA in RiskMetrics of J.P. Morgan (1994), the robust-EWMA in Guermat and Harris (2001), and the skewed-EWMA in Gerlach, Lu and Huang (2013). The EWMA is based on IGARCH model and RiskMetrics recommends the decay factor 0.94 for daily observations. For the robust-EWMA, Guermat and Harris (2001) found that a decay factor in the range $[0.92,0.95]$ performs well, so we use the same decay factor 0.94 as the EWMA. To implement the skewed-EWMA in Gerlach, Lu and Huang (2013), we use their procedure with daily re-estimated parameters, including both the time-varying parameters in the asymmetric-Laplace distribution and the time-independent decay factors. Figure 4 plots the sequential CVaR predictions using the aforementioned four methods: the semiparametric method with standard GARCH (as shown in Figure 2, EGARCH and GJR-GARCH lead to similar curves and are omitted), EWMA, robust-EWMA, and skewed-EWMA. The four methods lead to quite similar CVaR curves. 
Level $10 \%$
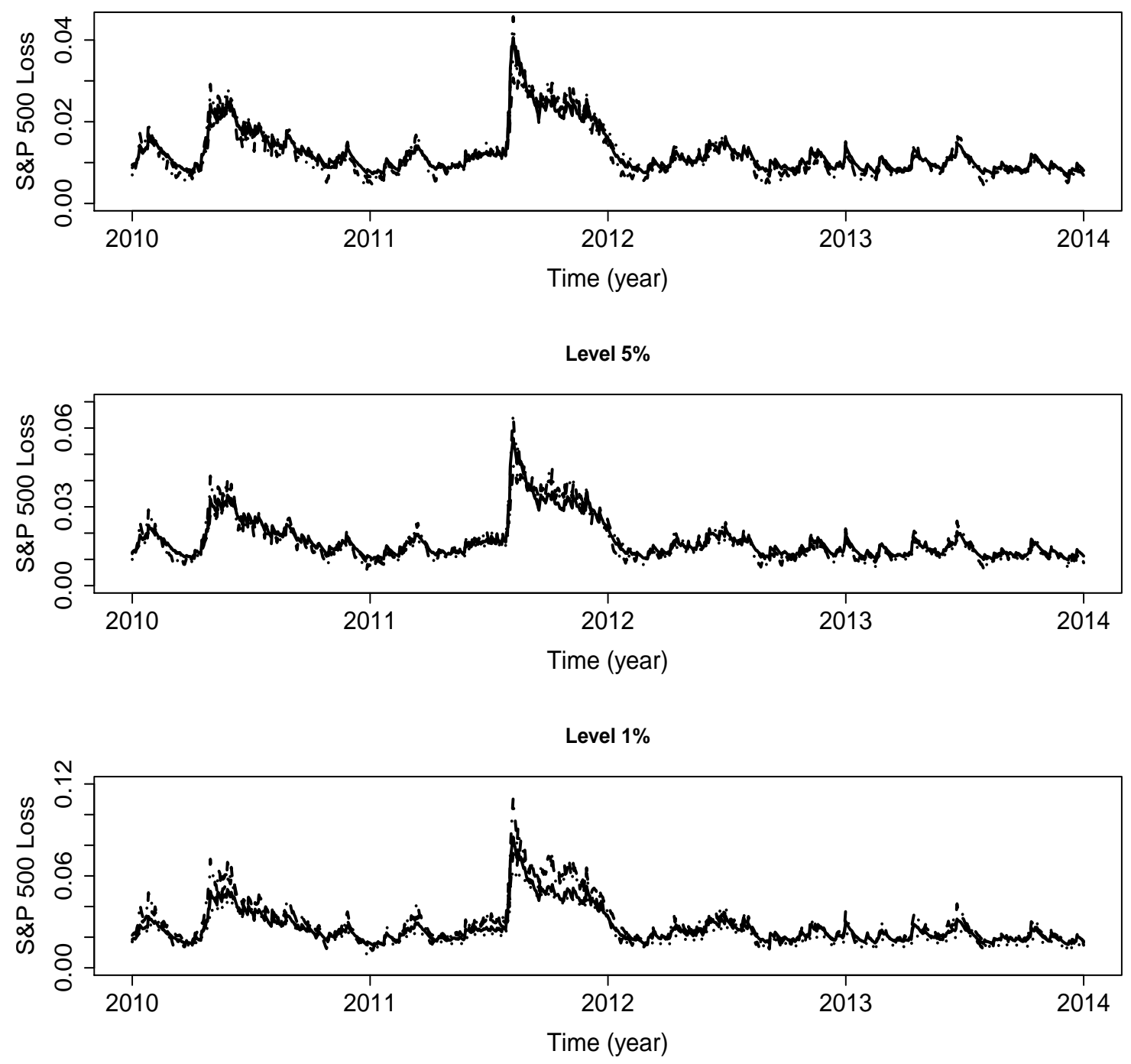

Figure 4: Comparison of sequentially predicted CVaR for daily losses during 2010-2013, using four methods: semiparametric method with standard GARCH (solid curve), the EWMA method (dotted curve), the robust-EWMA method (dashed curve), and the skewed-EWMA method (dotdashed curve). Top, middle, and bottom plots correspond to $1-\tau=10 \%, 5 \%, 1 \%$, respectively. 
To numerically examine the accuracy of the four methods in predicting CVaR $(1-$ $\left.\tau \mid Y_{j}, j \leq i-1\right)$ at times $n-999 \leq i \leq n$, first we consider the empirical violation rates (i.e., the empirical proportion that the observed loss exceeds the predicted CVaR):

$$
\frac{\text { the number of } n-999 \leq i \leq n \text { with } Y_{i} \geq \widehat{\mathrm{CVaR}}\left(1-\tau \mid Y_{j}, j \leq i-1\right)}{1000} \times 100 \% \text {. }
$$

Table 6 presents the empirical violation rates for the four methods at nominal levels $1-\tau=$ 10\%, 5\%, 1\%. We can see: (i) The semiparametric method with EGARCH and the skewedEWMA have comparable and top performance; (ii) The EWMA has the worst performance and substantially underestimates the risk at level $1 \%$ and $5 \%$, i.e., the empirical violation rate is much higher than the nominal level; and (iii) the other methods rank in the middle. For the semiparametric method, the empirical violation rates are generally quite close to the nominal levels. Therefore, although the true CVaR is unknown, we conclude that the predicted CVaR should be reasonably close to the true CVaR.

Table 6: Empirical violation rates for four methods: the proposed semiparametric method with different GARCH models (standard GARCH, EGARCH, GJR-GARCH), the EWMA method, the robust-EWMA method, and the skewed-EWMA method. The bracketed number (2.5\%) means that the violation rate is different from the nominal level, according to the unconditional coverage test at significance level $5 \%$.

\begin{tabular}{ll|ccc}
\hline & & \multicolumn{3}{|c}{ Level 1- } \\
method & & $1 \%$ & $5 \%$ & $10 \%$ \\
\hline semiparametric & standard GARCH & $1.3 \%$ & $5.0 \%$ & $9.0 \%$ \\
& EGARCH & $1.1 \%$ & $5.1 \%$ & $9.5 \%$ \\
& GJR-GARCH & $1.6 \%$ & $4.8 \%$ & $9.2 \%$ \\
\hline parametric & EWMA & $(2.5 \%)$ & $6.1 \%$ & $9.0 \%$ \\
& robust-EWMA & $0.8 \%$ & $5.6 \%$ & $9.7 \%$ \\
& skewed-EWMA & $1.1 \%$ & $5.6 \%$ & $10.0 \%$ \\
\hline
\end{tabular}

Next, as in Gerlach, Lu and Huang (2013), we consider two statistical tests:

- (Unconditional coverage test). Denote by $J_{v}$ the number of violations $Y_{i} \geq \widehat{\mathrm{CVaR}}(1-$ $\left.\tau \mid Y_{j}, j \leq i-1\right)$. Under the null hypothesis that the true violation rate is $1-\tau$ and the violations are independent, the binomial-distribution induced likelihood ratio test

$$
2\left[\left(J-J_{v}\right) \log \left(1-J_{v} / J\right)+J_{v} \log \left(J_{v} / J\right)-\left(J-J_{v}\right) \log (\tau)-J_{v} \log (1-\tau)\right]
$$


is asymptotically $\chi^{2}(1)$-distributed. In Table 6 , bracketed number indicates rejection of the null hypothesis at significance level 5\%. Thus, EWMA fails the test at nominal level $1-\tau=1 \%$ while other methods all pass the test.

- (Dynamic quantile test.) This test simultaneously tests the joint null hypothesis of correct violation rate and that the violations are uncorrelated over time. The idea is to regress the de-meaned "hit" variables on lagged "hit" variables and test for zero coefficients, i.e., testing for $\beta_{0}=\cdots=\beta_{p}=0$ in the linear regression

$$
\text { Hit }_{i} \sim \beta_{0}+\sum_{r=1}^{p} \beta_{r} \text { Hit }_{i-r}, \quad \text { Hit }_{i}:=\mathbf{1}_{Y_{i} \geq \widehat{\operatorname{CVaR}}\left(1-\tau \mid Y_{j}, j \leq i-1\right)}-(1-\tau) .
$$

Here $p$ is the order of the lagged regression. Denote by $Y$ and $X$ the corresponding response vector and covariate matrix of the above linear regression. Under the null hypothesis, $[\tau(1-\tau)]^{-1} Y^{T} X\left(X^{T} X\right)^{-1} X^{T} Y$ is asymptotically $\chi^{2}(p+1)$ distributed. See Engle and Manganelli (2004) for more details.

At significance level 5\%, Table 7 presents the results of the dynamic quantile test with different choices of order $p$ in (48), where "N" indicates rejection of the null hypothesis. The semiparametric method with EGARCH or GJR-GARCH and the skewed-EWMA have the best performance and pass the test for all choices of order $p=1,2,3,4$ and nominal levels $1-\tau=10 \%, 5 \%, 1 \%$, while the EWMA has the worst performance.

Combining the above analysis from violation rates, unconditional coverage test, and dynamic quantile test, we conclude that the semiparametric method with EGARCH and the skewed-EWMA have the best performance among the methods considered.

\subsection{Conditional VaR vs unconditional VaR}

Another popular approach is the unconditional or marginal VaR, which uses the quantile of the marginal distribution of the losses. To estimate unconditional VaR, one can use parametric or nonparametric methods. For parametric methods, one imposes some parametric distribution, denoted by $F_{\theta}(x)$, for the losses, then the $(1-\tau)$ unconditional $\operatorname{VaR}$ is $F_{\hat{\theta}}^{-1}(\tau)$, where $\hat{\theta}$ is an estimate of $\theta$. For example, a simple choice is the $N\left(\mu, \sigma^{2}\right)$ distribution with 
Table 7: Dynamic quantile test for the accuracy of the predicted $\widehat{\mathrm{CVaR}}\left(1-\tau \mid Y_{j}, j \leq i-1\right)$ at times $n-999 \leq i \leq n$ using the proposed semiparametric method with different GARCH models (standard GARCH, EGARCH, GJR-GARCH), the EWMA method, the robust-EWMA method, and the skewedEWMA method. "N" represents rejection of the joint null hypothesis of correct violation rate and that the violations are not correlated over time, at significance level 5\%.

\begin{tabular}{|c|c|c|c|c|c|c|c|c|c|c|c|c|c|}
\hline \multirow[b]{3}{*}{ method } & & \multicolumn{12}{|c|}{ Level $1-\tau$} \\
\hline & & \multicolumn{3}{|c|}{ order $p=1$} & \multicolumn{3}{|c|}{ order $p=2$} & \multicolumn{3}{|c|}{ order $p=3$} & \multicolumn{3}{|c|}{ order $p=4$} \\
\hline & & & $5 \%$ & $10 \%$ & $1 \%$ & $5 \%$ & $10 \%$ & $1 \%$ & $5 \%$ & $10 \%$ & $1 \%$ & $5 \%$ & $10 \%$ \\
\hline semiparametric & $\begin{array}{l}\text { standard GARCH } \\
\text { EGARCH } \\
\text { GJR-GARCH }\end{array}$ & & & & $\mathrm{N}$ & & & $\mathrm{N}$ & & & $\mathrm{N}$ & & \\
\hline parametric & $\begin{array}{l}\text { EWMA } \\
\text { robust-EWMA } \\
\text { skewed-EWMA }\end{array}$ & $\mathrm{N}$ & & & $\begin{array}{l}\mathrm{N} \\
\mathrm{N}\end{array}$ & & & $\begin{array}{l}\mathrm{N} \\
\mathrm{N}\end{array}$ & & & $\begin{array}{l}\mathrm{N} \\
\mathrm{N}\end{array}$ & & \\
\hline
\end{tabular}

$\mu$ an $\sigma^{2}$ estimated from the sample. For nonparametric or historical simulation method, one uses the sample $\tau$-th quantile of the losses to estimate the $(1-\tau)$ unconditional VaR.

Table 8 summarizes the empirical violation rates for the unconditional VaR using the aforementioned four methods. As in Table 6, the bracketed numbers indicate rejection of the null hypothesis, based on the unconditional coverage test at significance level $5 \%$. Comparing Table 6 and Table 8, we can clearly see the overall better performance of the conditional VaR over the unconditional VaR. Also, the dynamic quantile test (results not reported here) indicated that the unconditional VaR performs very poor. For example, at lagged order $p=2$, the dynamic quantile test rejects all the four unconditional VaR methods for all nominal levels $1-\tau=10 \%, 5 \%, 1 \%$. It is generally believed that financial returns are uncorrelated but not independent, thus the marginal distribution ignores such dependence and leads to inferior performance.

\section{Technical Conditions and Proofs}

Throughout $C_{1}, C_{2}, c, c_{1}, c_{2}, \ldots$, are generic constants that may vary from line to line. 
Table 8: Empirical violation rates for unconditional VaR using four methods: Normal distribution, Student- $t$ distribution, asymmetric Laplace distribution, and nonparametric estimate (historical simulation). The bracketed numbers mean that the violation rate is different from the nominal level, according to the unconditional coverage test at significance level $5 \%$.

\begin{tabular}{|c|c|c|c|}
\hline \multirow[b]{2}{*}{ method } & \multicolumn{3}{|c|}{ Level $1-\tau$} \\
\hline & $1 \%$ & $5 \%$ & $10 \%$ \\
\hline Normal & $0.9 \%$ & $(2.9 \%)$ & $(4.6 \%)$ \\
\hline Student- $t$ & $0.7 \%$ & $4.6 \%$ & $10.2 \%$ \\
\hline asymmetric-Laplace & $0.7 \%$ & $(3.7 \%)$ & $8.2 \%$ \\
\hline nonparametric estimate & $(0.4 \%)$ & $(3.5 \%)$ & $8.3 \%$ \\
\hline
\end{tabular}

\subsection{Technical conditions}

We list some technical conditions and preliminary results used in the proof of our theorems.

Assumption 3. In (4): (i) $\left\{\varepsilon_{i}\right\}_{i \in \mathbb{Z}}$ are i.i.d.. Denote by $\mathcal{F}_{i}$ the sigma-algebra generated by $\left\{\mathbf{X}_{i+1}, \mathbf{X}_{i}, \ldots ; \varepsilon_{i}, \varepsilon_{i-1}, \ldots\right\}$. For each $i, \varepsilon_{i}$ is independent of $\mathcal{F}_{i-1}$. (ii) $\left\{\left(\mathbf{X}_{i}, \varepsilon_{i}\right)\right\}_{i \in \mathbb{Z}}$ is stationary and $\alpha$-mixing with mixing coefficient $\alpha_{j} \leq C_{1} j^{-\alpha}$ for some constants $C_{1}>0$ and $\alpha>8+4 k$, where $k$ is the dimension of the parameter $\theta$.

Assumption 4. Recall $H\left(\theta, Y_{i}, \mathbf{X}_{i}\right)$ in (10). Let $\epsilon>0$ be some small constant. (i) $G(\theta, \varepsilon, x)$ is continuously differentiable in $\theta$ and $\varepsilon$. (ii) There exists $C_{2}>0$ such that $\mathbb{E}\left[\sup _{|\vartheta| \leq \epsilon, z \in \mathbb{R}} \mathbf{1}_{\left|H\left(\theta+\vartheta, Y_{0}, \mathbf{X}_{0}\right)-z\right| \leq v}\right] \leq C_{2} v$ for all $v>0$. (iii) Let $\dot{H}\left(\theta, Y_{i}, \mathbf{X}_{i}\right)$ be the partial derivative with respect to $\theta$. There exists $L\left(Y_{i}, \mathbf{X}_{i}\right)$,

$$
\sup _{|\vartheta| \leq \epsilon}\left|\dot{H}\left(\theta+\vartheta, Y_{i}, \mathbf{X}_{i}\right)\right| \leq L\left(Y_{i}, \mathbf{X}_{i}\right) \quad \text { and } \quad L\left(Y_{i}, \mathbf{X}_{i}\right) \in \mathcal{L}^{1}
$$

(iv) Define $J(\vartheta, z)=\mathbb{P}\left\{H\left(\theta+\vartheta, Y_{0}, \mathbf{X}_{0}\right) \leq z\right\}, \vartheta \in \mathbb{R}^{k}, z \in \mathbb{R}$. Let $\dot{J}(\vartheta, z)$ and $\ddot{J}(\vartheta, z)$ be the gradient vector and Hessian matrix of $J(\vartheta, z)$ with respect to $\vartheta$. $\dot{J}(0, z)$ is continuous in $z$ and $|\ddot{J}(\vartheta, z)|$ is bounded on $|\vartheta| \leq \epsilon, z \in \mathbb{R}$.

Intuitively, Assumption 4(ii) asserts that the probability mass of $H\left(\theta+\vartheta, Y_{0}, \mathbf{X}_{0}\right)$ on $[z-v, z+v]$ is $O(v)$ uniformly, which is reasonable if the density is uniformly bounded. Assumption 4(iii) is used to control errors in Taylor's expansions. In view of $\hat{\varepsilon}_{i}=H\left(\hat{\theta}, Y_{i}, \mathbf{X}_{i}\right)$ in (11), $J(\vartheta, z)$ in Assumption 4(iv) measures how the distribution function of $\hat{\varepsilon}_{i}$ changes in response to the departure $\vartheta$ from the true parameter $\theta$. In particular, $J(0, z)=\mathbb{P}\left\{\varepsilon_{0} \leq z\right\}$. 
Assumption 5. Density $f_{\varepsilon}(z)$ of $\varepsilon_{i}$ is continuous, bounded, positive at $z=Q_{\varepsilon}(\tau)$.

Assumption 5*. Density $f_{\varepsilon}(z)$ of $\varepsilon_{i}$ is continuous, bounded, positive on $\left[Q_{\varepsilon}(\delta), Q_{\varepsilon}(1-\delta)\right]$.

Definition 1. Recall $\mathcal{F}_{i}$ in Assumption 3. We say that a function $g\left(z, \mathcal{F}_{i}\right)$ (may be vector or matrix valued) is stochastically continuous at a point $z^{*}$ if

$$
\lim _{\epsilon \rightarrow 0} \mathbb{E}\left[\mathcal{G}\left(\epsilon, \mathcal{F}_{i}\right)\right]=0, \quad \text { where } \mathcal{G}\left(\epsilon, \mathcal{F}_{i}\right)=\sup _{\left|z-z^{*}\right| \leq \epsilon}\left|g\left(z, \mathcal{F}_{i}\right)-g\left(z^{*}, \mathcal{F}_{i}\right)\right|
$$

In $(50), \mathcal{G}\left(\epsilon, \mathcal{F}_{i}\right)$ quantifies the maximal fluctuation of $g\left(z, \mathcal{F}_{i}\right)$ in the $\epsilon$-neighborhood of $z^{*}$, and the condition $\lim _{\epsilon \rightarrow 0} \mathbb{E}\left[\mathcal{G}\left(\epsilon, \mathcal{F}_{i}\right)\right]=0$ asserts that the maximal fluctuation asymptotically vanishes under expectation, which suggests "stochastic continuity". The stochastic continuity extends the continuity of deterministic functions to that of stochastic functions.

Assumption 6. $\dot{G}_{\theta}\left(\theta, \varepsilon, \mathbf{X}_{i}\right) / \dot{G}_{\varepsilon}\left(\theta, \varepsilon, \mathbf{X}_{i}\right)$ is stochastically continuous at $\left(\theta, Q_{\varepsilon}(\tau)\right)$. For $H\left(\theta, Y_{i}, \mathbf{X}_{i}\right)$ in (10) and $D\left(\theta, \varepsilon_{i}, \mathbf{X}_{i}\right)$ in Assumption 2 , write $D_{i}(\vartheta)=D\left(\vartheta, H\left(\vartheta, Y_{i}, \mathbf{X}_{i}\right), \mathbf{X}_{i}\right)$. Suppose that $D_{i}(\vartheta)$ and $D_{i}(\vartheta) D_{i}(\vartheta)^{T}$ are stochastically continuous at $\vartheta=\theta$.

\subsection{Some lemmas}

Here we list some useful lemmas whose proofs can be found in the Appendix of the full paper, available on the corresponding author's webpage.

Lemma 1. Suppose that $g\left(z, \mathcal{F}_{i}\right)$ is stochastically continuous at $z=z^{*}$ and $g\left(z^{*}, \mathcal{F}_{i}\right) \in \mathcal{L}^{1}$. Then for any random sequence $z_{n} \stackrel{p}{\rightarrow} z^{*}, n^{-1} \sum_{i=1}^{n} g\left(z_{n}, \mathcal{F}_{i}\right)=\mathbb{E}\left[g\left(z^{*}, \mathcal{F}_{0}\right)\right]+o_{p}(1)$.

Lemma 2. Let $z, z^{\prime} \in \mathbb{R}$. Then for any $c>0,\left|\mathbf{1}_{z \leq 0}-\mathbf{1}_{z^{\prime} \leq 0}\right| \leq 2 \mathbf{1}_{\left|z-z^{\prime}\right| \geq c}+\mathbf{1}_{\left|z^{\prime}\right|<c}$.

Lemma 3. let $\left\{\xi_{i}\right\}_{i \in \mathbb{Z}}$ be a stationary $\alpha$-mixing process with mixing coefficient $\alpha_{j}, j \in \mathbb{N}$. Assume $\mathbb{E}\left(\xi_{0}\right)=0$ and $\left|\xi_{i}\right| \leq c$ for some $c$. Then, for $\ell=1, \ldots,\lfloor n / 2\rfloor$ and $z>0$,

$$
\mathbb{P}\left\{\left|\sum_{i=1}^{n} \xi_{i}\right|>z\right\} \leq 4 \exp \left\{-\frac{z^{2} \ell}{144 n^{2} \mathbb{E}\left(\xi_{0}^{2}\right)+4 c z n}\right\}+22 \ell \alpha_{\lfloor n /(2 \ell)\rfloor} \sqrt{1+\frac{4 c n}{z}}
$$

Lemma 4. Recall $J(\vartheta, z)$ and $\dot{J}(\vartheta, z)$ in Assumption 4(iv). Then

$$
\dot{J}(0, z)=f_{\varepsilon}(z) \mathbb{E}\left[\frac{\dot{G}_{\theta}\left(\theta, z, \mathbf{X}_{0}\right)}{\dot{G}_{\varepsilon}\left(\theta, z, \mathbf{X}_{0}\right)}\right] .
$$


Lemma 5. Let $d(\cdot, \cdot)$ be a measurable function such that $d\left(\varepsilon_{i}, \mathbf{X}_{i}\right) \in \mathcal{L}^{2}$ and $\mathbb{E}\left[d\left(\varepsilon_{i}, \mathbf{X}_{i}\right) \mid \mathbf{X}_{i}\right]=$ 0. Suppose Assumption 3 holds. Then $n^{-1 / 2} \sum_{i=1}^{n} d\left(\varepsilon_{i}, \mathbf{X}_{i}\right) \Rightarrow N\left(0, \mathbb{E}\left[d^{2}\left(\varepsilon_{0}, \mathbf{X}_{0}\right)\right]\right)$.

Lemma 6. Recall $\hat{f}_{\varepsilon}(z)$ in (18). Under the conditions in Theorem $3, \hat{f}_{\varepsilon}(z) \stackrel{p}{\rightarrow} f_{\varepsilon}(z)$ uniformly in the neighborhood of $z=Q_{\varepsilon}(\tau)$.

Lemma 7. Under the conditions in Theorem 3, we have $n^{-1} \sum_{i=1}^{n}\left[\tau-\mathbf{1}_{\hat{\varepsilon}_{i}<\hat{Q}_{\varepsilon}(\tau)}\right] \stackrel{p}{\rightarrow} 0$.

Lemma 8. Suppose that Assumption $5^{*}$ holds. Let $\Delta$ be any given number. Define

$$
I(z)=\sum_{i=1}^{n} \zeta_{i}(z), \quad \zeta_{i}(z)=\int_{0}^{\frac{\Delta}{\sqrt{n}}}\left\{\left[\mathbf{1}_{\varepsilon_{i} \leq z+s}-\mathbf{1}_{\varepsilon_{i} \leq z}\right]-\left[F_{\varepsilon}(z+s)-F_{\varepsilon}(z)\right]\right\} d s .
$$

Then $I(z)=o_{p}(1)$ uniformly on $z \in\left[Q_{\varepsilon}(\delta), Q_{\varepsilon}(1-\delta)\right]$.

Lemma 9. Assume that $f_{\varepsilon}$ is continuous. Then for any given constants $c_{1}, c_{2}>0$,

$$
\sup _{|z| \leq c_{1},|v| \leq c_{2} / \sqrt{n}}\left|\frac{1}{n} \sum_{i=1}^{n} \mathbf{1}_{\varepsilon_{i} \leq z+v}-\frac{1}{n} \sum_{i=1}^{n} \mathbf{1}_{\varepsilon_{i} \leq z}-v f_{\varepsilon}(z)\right|=o_{p}\left(n^{-1 / 2}\right) .
$$

\subsection{Proof of main theorems}

Proof of Theorem 1. To reflect the dependence of $\hat{F}_{\varepsilon}(z)$ on $\hat{\theta}$ and in view of $\hat{\varepsilon}_{i}=$ $H\left(\hat{\theta}, Y_{i}, \mathbf{X}_{i}\right)$, write $\vartheta$ as the departure from the true parameter $\theta$ and define

$$
\hat{F}_{\varepsilon}(\vartheta, z)=\frac{1}{n} \sum_{i=1}^{n} \mathbf{1}_{H\left(\theta+\vartheta, Y_{i}, \mathbf{X}_{i}\right) \leq z}
$$

Then $\hat{F}_{\varepsilon}(z)=\hat{F}_{\varepsilon}(\hat{\theta}-\theta, z)$. By the expression for $\dot{J}(0, z)$ in (52) and the assumption $\hat{\theta}-\theta=O_{p}\left(n^{-1 / 2}\right)$, in order to prove (14), it suffices to prove that, for all given $c_{1}>0$,

$$
\sup _{|\vartheta| \leq c_{1} / \sqrt{n},|z| \leq c}\left|\hat{F}_{\varepsilon}(\vartheta, z)-\hat{F}_{\varepsilon}(0, z)-\dot{J}(0, z)^{T} \vartheta\right|=o_{p}\left(n^{-1 / 2}\right) .
$$

For simplicity we assume that: (i) $k=1$, i.e., $\vartheta$ is scalar-valued, (ii) $c_{1}=c=1$, and (iii) $\vartheta \in[0,1 / \sqrt{n}]$ and $z \in[0,1]$, since the general $k$-dimensional case follows similarly. Let

$$
\xi_{i}(\vartheta, z)=\mathbf{1}_{H\left(\theta+\vartheta, Y_{i}, \mathbf{X}_{i}\right) \leq z}-\mathbf{1}_{H\left(\theta, Y_{i}, \mathbf{X}_{i}\right) \leq z}
$$


Recall $J(\vartheta, z)$ in Assumption 4(iv). By Taylor's expansion, $\mathbb{E}\left[\xi_{i}(\vartheta, z)\right]=J(\vartheta, z)-J(0, z)=$ $\dot{J}(0, z)^{T} \vartheta+O\left(n^{-1}\right)$ uniformly on $\vartheta \in[0,1 / \sqrt{n}], z \in[0,1]$. To prove (54), it suffices to show

$$
\sup _{(\vartheta, z) \in[0,1 / \sqrt{n}] \times[0,1]}|M(\vartheta, z)|=o_{p}(\sqrt{n}), \quad \text { where } \quad M(\vartheta, z)=\sum_{i=1}^{n}\left\{\xi_{i}(\vartheta, z)-\mathbb{E}\left[\xi_{i}(\vartheta, z)\right]\right\} .
$$

To prove (56), we adopt a chain argument. Let $N=\left\lfloor n^{1+\epsilon}\right\rfloor$ with $\epsilon>0$ to be determined later, and consider the evenly spaced points

$$
\vartheta_{j}=j \omega_{1} \quad \text { with } \quad \omega_{1}=\frac{1}{\sqrt{n} N}, \quad z_{j}=j \omega_{2} \quad \text { with } \quad \omega_{2}=\frac{1}{N}, \quad j=0,1, \ldots, N .
$$

The $(N+1)^{2}$ grid points $\left\{\left(\vartheta_{j}, z_{j^{\prime}}\right)\right\}_{j, j^{\prime}=0}^{N}$ partition $[0,1 / \sqrt{n}] \times[0,1]$ into $N^{2}$ cells. For each $(\vartheta, z) \in[0,1 / \sqrt{n}] \times[0,1]$, there exists one grid point $\left(\vartheta_{j}, z_{j^{\prime}}\right)$ such that $\left|\vartheta-\vartheta_{j}\right| \leq \omega_{1}$ and $\left|z-z_{j^{\prime}}\right| \leq \omega_{2}$. Thus, by $|M(\vartheta, z)| \leq\left|M\left(\vartheta_{j}, z_{j^{\prime}}\right)\right|+\left|M(\vartheta, z)-M\left(\vartheta_{j}, z_{j^{\prime}}\right)\right|$, we have

$$
\sup _{(\vartheta, z) \in[0,1 / \sqrt{n}] \times[0,1]}|M(\vartheta, z)| \leq \max _{0 \leq j, j^{\prime} \leq N}\left|M\left(\vartheta_{j}, z_{j^{\prime}}\right)\right|+R_{n}
$$

where

$$
R_{n}=\sup _{\Omega}\left|M(\vartheta, z)-M\left(\vartheta^{\prime}, z^{\prime}\right)\right| \quad \text { with } \quad \Omega=\left\{\left|\vartheta-\vartheta^{\prime}\right| \leq \omega_{1},\left|z-z^{\prime}\right| \leq \omega_{2}\right\}
$$

It is easy to see that

$$
\sup _{\Omega}\left|\xi_{i}(\vartheta, z)-\xi_{i}\left(\vartheta^{\prime}, z^{\prime}\right)\right| \leq 2 \sup _{\Omega}\left[\left|\mathbf{1}_{H\left(\theta+\vartheta, Y_{i}, \mathbf{X}_{i}\right) \leq z}-\mathbf{1}_{H\left(\theta+\vartheta^{\prime}, Y_{i}, \mathbf{X}_{i}\right) \leq z^{\prime}}\right|\right] .
$$

Therefore, by (56), (58) and (59),

$$
\begin{aligned}
\mathbb{E}\left(R_{n}\right) & \leq 4 n \mathbb{E}\left\{\sup _{\Omega}\left[\left|\mathbf{1}_{U(\vartheta)-z \leq 0}-\mathbf{1}_{U\left(\vartheta^{\prime}\right)-z^{\prime} \leq 0}\right|\right]\right\} \text { with } U(\vartheta)=H\left(\theta+\vartheta, Y_{0}, \mathbf{X}_{0}\right) \\
& \leq 4 n \mathbb{E}\left\{\sup _{\Omega}\left[2 \mathbf{1}_{\left|\left[U(\vartheta)-U\left(\vartheta^{\prime}\right)\right]-\left(z-z^{\prime}\right)\right| \geq \lambda}+\mathbf{1}_{|U(\vartheta)-z|<\lambda}\right]\right\}
\end{aligned}
$$

where the second " $\leq$ " follows from Lemma 2 and $\lambda>0$ is any given number. By Assumption 4(iii), on $\Omega$, $\left|\left[U(\vartheta)-U\left(\vartheta^{\prime}\right)\right]-\left(z-z^{\prime}\right)\right| \leq \omega_{1} L\left(Y_{0}, \mathbf{X}_{0}\right)+\omega_{2}$. Thus, by (60),

$$
\begin{aligned}
\mathbb{E}\left(R_{n}\right) & \leq 8 n \mathbb{P}\left\{\omega_{1} L\left(Y_{0}, \mathbf{X}_{0}\right)+\omega_{2} \geq \lambda\right\}+4 n \mathbb{E}\left\{\sup _{\Omega} \mathbf{1}_{|U(\vartheta)-z|<\lambda}\right\} \\
& \leq 8 n \frac{\omega_{1} \mathbb{E}\left|L\left(Y_{0}, \mathbf{X}_{0}\right)\right|+\omega_{2}}{\lambda}+O(n \lambda)
\end{aligned}
$$


where the second " $\leq$ " follows from Markov inequality and Assumption 4(ii). Letting $\lambda=\sqrt{\omega_{2}}$, we have $R_{n}=O_{p}\left(n \sqrt{\omega_{2}}\right)=o_{p}(\sqrt{n})$.

By (57), it remains to prove $\max _{0 \leq j, j^{\prime} \leq N}\left|M\left(\vartheta_{j}, z_{j^{\prime}}\right)\right|=o_{p}(\sqrt{n})$. Note that $\xi_{1}^{2}(\vartheta, z)=$ $\left|\xi_{1}(\vartheta, z)\right|=\left|\mathbf{1}_{U(\vartheta)-z \leq 0}-\mathbf{1}_{U(0)-z \leq 0}\right|$ with $U(\vartheta)$ defined in (60). Therefore, by Lemma 2 ,

$$
\operatorname{var}\left\{\xi_{1}(\vartheta, z)\right\} \leq \mathbb{E}\left|\mathbf{1}_{U(\vartheta)-z \leq 0}-\mathbf{1}_{U(0)-z \leq 0}\right| \leq \mathbb{E}\left[2 \mathbf{1}_{|U(\vartheta)-U(0)| \geq n^{-1 / 4}}+\mathbf{1}_{|U(\vartheta)-z|<n^{-1 / 4}}\right] .
$$

By Assumption 4(iii), $|U(\vartheta)-U(0)| \leq n^{-1 / 2} L\left(Y_{0}, \mathbf{X}_{0}\right)$ on $|\vartheta| \leq 1 / \sqrt{n}$. Applying the latter inequality to (62) and by the same argument in (61) [i.e., Markov inequality and Assumption 4(ii)], we obtain that, there exists some constant $c_{2}$ such that

$$
\operatorname{var}\left\{\xi_{1}(\vartheta, z)\right\} \leq c_{2} n^{-1 / 4}, \quad \text { uniformly on }|\vartheta| \leq 1 / \sqrt{n}, z \in \mathbb{R} \text {. }
$$

Note that $\left|\xi_{i}(\vartheta, z)-\mathbb{E}\left[\xi_{i}(\vartheta, z)\right]\right| \leq 2$. By Lemma 3 , for any $c_{3}>0$ and $\ell=1, \ldots,\lfloor n / 2\rfloor$,

$$
\mathbb{P}\left\{|M(\vartheta, z)| \geq c_{3} \sqrt{n}\right\} \leq 4 \exp \left(-\frac{c_{3}^{2} n \ell}{144 c_{2} n^{7 / 4}+8 c_{3} n^{3 / 2}}\right)+22 \ell \alpha_{\lfloor n / \ell\rfloor} \sqrt{1+\frac{8 n}{c_{3} \sqrt{n}}} .
$$

Let $\ell=\left\lfloor n^{\beta}\right\rfloor$ with some $\beta \in(3 / 4,1)$ to be determined later. Recall $\alpha_{j} \leq C_{1} j^{-\alpha}$ in Assumption 3(ii). Thus, from (64), there exists constants $c_{4}$ and $c_{5}$ such that

$$
\mathbb{P}\left\{|M(\vartheta, z)| \geq c_{3} \sqrt{n}\right\} \leq c_{5}\left[\exp \left\{-c_{4} n^{\beta-\frac{3}{4}}\right\}+n^{\beta(1+\alpha)+\frac{1}{4}-\alpha}\right]
$$

uniformly over $|\vartheta| \leq 1 / \sqrt{n}, z \in \mathbb{R}$. Recall that $N=\left\lfloor n^{1+\epsilon}\right\rfloor$. By (65),

$$
\begin{aligned}
\mathbb{P}\left\{\sup _{0 \leq j, j^{\prime} \leq N}\left|M\left(\vartheta_{j}, z_{j^{\prime}}\right)\right| \geq c_{3} \sqrt{n}\right\} & \leq \sum_{j, j^{\prime}=0}^{N} \mathbb{P}\left\{\left|M\left(\vartheta_{j}, z_{j^{\prime}}\right)\right| \geq c_{3} \sqrt{n}\right\} \\
& =O\left[n^{2+2 \epsilon} \exp \left\{-c_{4} n^{\beta-\frac{3}{4}}\right\}+n^{2 \epsilon+\frac{9}{4}+\beta(1+\alpha)-\alpha}\right] .
\end{aligned}
$$

From Assumption 3(ii), $\alpha>12(k=1)$, which implies $3 / 4<(\alpha-9 / 4) /(1+\alpha)$. Take any

$$
\frac{3}{4}<\beta<\frac{\alpha-9 / 4}{1+\alpha} \quad \text { and } \quad \epsilon=\frac{\alpha-9 / 4-\beta(1+\alpha)}{3}>0 .
$$

Then it is easy to see that the right hand side of (66) goes to zero. Since $c_{3}$ is arbitrary, we conclude $\sup _{0 \leq j, j^{\prime} \leq N}\left|M\left(\vartheta_{j}, z_{j^{\prime}}\right)\right|=o_{p}(\sqrt{n})$. This completes the proof.

Proof of Theorem 2. In what follows we shall prove only the CLT in (ii) under Assumption 2. The same argument can be used to prove the weaker assertion $\hat{Q}(\tau \mid x)=$ $Q(\tau \mid x)+O_{p}\left(n^{-1 / 2}\right)$ in (i) under the weaker condition $\hat{\theta}=\theta+O_{p}\left(n^{-1 / 2}\right)$. 
The empirical quantile function $\hat{Q}_{\varepsilon}(\tau)$ is a solution to

$$
\min _{\nu} \sum_{i=1}^{n} \rho_{\tau}\left(\hat{\varepsilon}_{i}-\nu\right), \quad \rho_{\tau}(v)=v\left(\tau-\mathbf{1}_{v \leq 0}\right)
$$

Thus, $\hat{Q}_{\varepsilon}(\tau)$ is also a minimizer of the function $\sum_{i=1}^{n}\left[\rho_{\tau}\left(\hat{\varepsilon}_{i}-\nu\right)-\rho_{\tau}\left(\hat{\varepsilon}_{i}-Q_{\varepsilon}(\tau)\right)\right]$ of $\nu$. Let the transformation $\Delta=\sqrt{n}\left[\nu-Q_{\varepsilon}(\tau)\right]$. Then $\hat{\varepsilon}_{i}-\nu=\hat{\varepsilon}_{i}-Q_{\varepsilon}(\tau)-\Delta / \sqrt{n}$. Thus, $\hat{\Delta}:=\sqrt{n}\left[\hat{Q}_{\varepsilon}(\tau)-Q_{\varepsilon}(\tau)\right]$ is a minimizer of the re-parametrized function $S(\Delta)$ :

$$
S(\Delta)=\sum_{i=1}^{n}\left[\rho_{\tau}\left\{\hat{\varepsilon}_{i}-Q_{\varepsilon}(\tau)-\Delta / \sqrt{n}\right\}-\rho_{\tau}\left\{\hat{\varepsilon}_{i}-Q_{\varepsilon}(\tau)\right\}\right] .
$$

By Knight's identity $\rho_{\tau}(u-v)-\rho_{\tau}(u)=-v\left(\tau-\mathbf{1}_{u<0}\right)+\int_{0}^{v}\left(\mathbf{1}_{u \leq s}-\mathbf{1}_{u \leq 0}\right) d s$, we can rewrite

$$
S(\Delta)=-\frac{\Delta}{\sqrt{n}} \sum_{i=1}^{n}\left[\tau-\mathbf{1}_{\hat{\varepsilon}_{i}<Q_{\varepsilon}(\tau)}\right]+\int_{0}^{\frac{\Delta}{\sqrt{n}}} \sum_{i=1}^{n}\left[\mathbf{1}_{\hat{\varepsilon}_{i} \leq Q_{\varepsilon}(\tau)+s}-\mathbf{1}_{\hat{\varepsilon}_{i} \leq Q_{\varepsilon}(\tau)}\right] d s .
$$

For the second term, by the uniform approximation of $\hat{F}_{\varepsilon}(z)$ in Theorem 1 , for fixed $\Delta$,

$$
\int_{0}^{\frac{\Delta}{\sqrt{n}}} \sum_{i=1}^{n}\left[\mathbf{1}_{\hat{\varepsilon}_{i} \leq Q_{\varepsilon}(\tau)+s}-\mathbf{1}_{\hat{\varepsilon}_{i} \leq Q_{\varepsilon}(\tau)}\right] d s=I_{1}+I_{2}+o_{p}(1),
$$

where

$$
\begin{aligned}
& I_{1}=\sum_{i=1}^{n} \int_{0}^{\frac{\Delta}{\sqrt{n}}}\left[\mathbf{1}_{\varepsilon_{i} \leq Q_{\varepsilon}(\tau)+s}-\mathbf{1}_{\varepsilon_{i} \leq Q_{\varepsilon}(\tau)}\right] d s, \\
& I_{2}=n \int_{0}^{\frac{\Delta}{\sqrt{n}}}\left[\dot{J}\left(0, Q_{\varepsilon}(\tau)+s\right)-\dot{J}\left(0, Q_{\varepsilon}(\tau)\right)\right]^{T}(\hat{\theta}-\theta) d s .
\end{aligned}
$$

For $I_{1}$, we have

$$
\mathbb{E}\left(I_{1}\right)=n \int_{0}^{\frac{\Delta}{\sqrt{n}}}\left[F_{\varepsilon}\left(Q_{\varepsilon}(\tau)+s\right)-F_{\varepsilon}\left(Q_{\varepsilon}(\tau)\right)\right] d s \quad \rightarrow \quad \frac{f_{\varepsilon}\left(Q_{\varepsilon}(\tau)\right)}{2} \Delta^{2},
$$

in view of $F_{\varepsilon}\left(Q_{\varepsilon}(\tau)+s\right)-F_{\varepsilon}\left(Q_{\varepsilon}(\tau)\right)=s f_{\varepsilon}\left(Q_{\varepsilon}(\tau)\right)+o(s)$. Also, $\left\{\varepsilon_{i}\right\}$ are i.i.d., so

$$
\begin{aligned}
\operatorname{var}\left(I_{1}\right) & =\sum_{i=1}^{n} \operatorname{var}\left\{\int_{0}^{\frac{\Delta}{\sqrt{n}}}\left[\mathbf{1}_{\varepsilon_{i} \leq Q_{\varepsilon}(\tau)+s}-\mathbf{1}_{\varepsilon_{i} \leq Q_{\varepsilon}(\tau)}\right] d s\right\} \\
& \leq \sum_{i=1}^{n} \mathbb{E}\left\{\left[\frac{\Delta}{\sqrt{n}} \mathbf{1}_{\left|\varepsilon_{i}-Q_{\varepsilon}(\tau)\right| \leq|\Delta| / \sqrt{n}}\right]^{2}\right\}=\Delta^{2} \mathbb{E} \mathbf{1}_{\left|\varepsilon_{0}-Q_{\varepsilon}(\tau)\right| \leq|\Delta| / \sqrt{n}} \rightarrow
\end{aligned}
$$


From (71)-(72), $I_{1}=\Delta^{2} f_{\varepsilon}\left(Q_{\varepsilon}(\tau)\right) / 2+o_{p}(1)$. From $\hat{\theta}-\theta=O_{p}\left(n^{-1 / 2}\right)$ and the continuity of $\dot{J}(0, z), I_{2}=o_{p}(1)$ for each fixed $\Delta$. Therefore, in view of (67)-(68), for each fixed $\Delta$,

$$
S(\Delta)=\tilde{S}(\Delta)+o_{p}(1), \quad \tilde{S}(\Delta)=-\frac{\Delta}{\sqrt{n}} \sum_{i=1}^{n}\left[\tau-\mathbf{1}_{\hat{\varepsilon}_{i}<Q_{\varepsilon}(\tau)}\right]+\frac{f_{\varepsilon}\left(Q_{\varepsilon}(\tau)\right)}{2} \Delta^{2} .
$$

By the quadratic approximation and convexity lemma [Pollard (1991)], the minimizer $\hat{\Delta}=\sqrt{n}\left[\hat{Q}_{\varepsilon}(\tau)-Q_{\varepsilon}(\tau)\right]$ of $S(\Delta)$ has the approximation

$$
\hat{\Delta}=\underset{\Delta}{\operatorname{argmin}} \tilde{S}(\Delta)+o_{p}(1)=\frac{1}{\sqrt{n} f_{\varepsilon}\left(Q_{\varepsilon}(\tau)\right)} \sum_{i=1}^{n}\left[\tau-\mathbf{1}_{\hat{\varepsilon}_{i}<Q_{\varepsilon}(\tau)}\right]+o_{p}(1) .
$$

Recall that $\hat{\Delta}=\sqrt{n}\left[\hat{Q}_{\varepsilon}(\tau)-Q_{\varepsilon}(\tau)\right]$. In (74), by the uniform approximation for $\sum_{i=1}^{n} \mathbf{1}_{\hat{\varepsilon}_{i}<Q_{\varepsilon}(\tau)}$ in Theorem 1 and the Bahadur representation for $\hat{\theta}-\theta$ in Assumption 2, we obtain

$$
\hat{Q}_{\varepsilon}(\tau)-Q_{\varepsilon}(\tau)=\frac{1}{n} \sum_{i=1}^{n}\left\{\frac{\tau-\mathbf{1}_{\varepsilon_{i}<Q_{\varepsilon}(\tau)}}{f_{\varepsilon}\left(Q_{\varepsilon}(\tau)\right)}-\mathbb{E}\left[\frac{\dot{G}_{\theta}\left(\theta, Q_{\varepsilon}(\tau), \mathbf{X}_{0}\right)}{\dot{G}_{\varepsilon}\left(\theta, Q_{\varepsilon}(\tau), \mathbf{X}_{0}\right)}\right]^{T} D\left(\theta, \varepsilon_{i}, \mathbf{X}_{i}\right)\right\}+o_{p}\left(n^{-1 / 2}\right)
$$

By Lemma 5, $\hat{Q}_{\varepsilon}(\tau)=Q_{\varepsilon}(\tau)+O_{p}\left(n^{-1 / 2}\right)$. Thus, by (75) and Assumptions 2 and 4(i),

$$
\begin{aligned}
& G\left(\hat{\theta}, \hat{Q}_{\varepsilon}(\tau), x\right)-G\left(\theta, Q_{\varepsilon}(\tau), x\right) \\
= & \dot{G}_{\theta}\left(\theta, Q_{\varepsilon}(\tau), x\right)^{T}(\hat{\theta}-\theta)+\dot{G}_{\varepsilon}\left(\theta, Q_{\varepsilon}(\tau), x\right)\left[\hat{Q}_{\varepsilon}(\tau)-Q_{\varepsilon}(\tau)\right]+o_{p}\left(n^{-1 / 2}\right) \\
= & \dot{G}_{\varepsilon}\left(x, Q_{\varepsilon}(\tau), x\right) \frac{1}{n} \sum_{i=1}^{n} W_{i}(\tau)+o_{p}\left(n^{-1 / 2}\right),
\end{aligned}
$$

where $W_{i}(\tau)$ is defined in (17). The result then follows from (76) and Lemma 5.

Proof of Theorem 3. First, we prove $\bar{W}(\tau)=o_{p}(1)$. Since $\hat{\theta} \stackrel{p}{\rightarrow} \theta$ and $\hat{Q}_{\varepsilon}(\tau) \stackrel{p}{\rightarrow} Q_{\varepsilon}(\tau)$, by the continuity of $\dot{G}_{\theta}(\theta, \varepsilon, x)$ and $\dot{G}_{\varepsilon}(\theta, \varepsilon, x)$ [Assumption 4(i)] and the stochastic continuity condition in Assumption 6, from Lemma 1 we obtain

$$
\begin{gathered}
\frac{\dot{G}_{\theta}\left(\hat{\theta}, \hat{Q}_{\varepsilon}(\tau), x\right)}{\dot{G}_{\varepsilon}\left(\hat{\theta}, \hat{Q}_{\varepsilon}(\tau), x\right)}-\frac{1}{n} \sum_{i=1}^{n} \frac{\dot{G}_{\theta}\left(\hat{\theta}, \hat{Q}_{\varepsilon}(\tau), \mathbf{X}_{i}\right)}{\dot{G}_{\varepsilon}\left(\hat{\theta}, \hat{Q}_{\varepsilon}(\tau), \mathbf{X}_{i}\right)} \stackrel{p}{\rightarrow} \frac{\dot{G}_{\theta}\left(\theta, Q_{\varepsilon}(\tau), x\right)}{\dot{G}_{\varepsilon}\left(\theta, Q_{\varepsilon}(\tau), x\right)}-\mathbb{E}\left[\frac{\dot{G}_{\theta}\left(\theta, Q_{\varepsilon}(\tau), \mathbf{X}_{0}\right)}{\dot{G}_{\varepsilon}\left(\theta, Q_{\varepsilon}(\tau), \mathbf{X}_{0}\right)}\right] \\
\frac{1}{n} \sum_{i=1}^{n} D\left(\hat{\theta}, \hat{\varepsilon}_{i}, \mathbf{X}_{i}\right)=\frac{1}{n} \sum_{i=1}^{n} D\left(\hat{\theta}, H\left(\hat{\theta}, Y_{i}, \mathbf{X}_{i}\right), \mathbf{X}_{i}\right) \stackrel{p}{\rightarrow} \mathbb{E}\left[D\left(\theta, \varepsilon_{0}, \mathbf{X}_{0}\right)\right]=0 .
\end{gathered}
$$

By $(77)-(78)$ and Lemmas $6-7, \bar{W}(\tau)=o_{p}(1)$. 
It remains to prove $n^{-1} \sum_{i=1}^{n} \widehat{W}_{i}(\tau)^{2} \stackrel{p}{\rightarrow} \mathbb{E}\left[W_{1}(\tau)^{2}\right]$. Recall $D_{i}(\vartheta)=D\left(\vartheta, H\left(\vartheta, Y_{i}, \mathbf{X}_{i}\right), \mathbf{X}_{i}\right)$ in Assumption 6. Then $D\left(\hat{\theta}, \hat{\varepsilon}_{i}, \mathbf{X}_{i}\right)=D_{i}(\hat{\theta})$ and $D\left(\theta, \varepsilon_{i}, \mathbf{X}_{i}\right)=D_{i}(\theta)$. By Lemma 6 and (77), it suffices to prove

$$
\begin{aligned}
& \frac{1}{n} \sum_{i=1}^{n}\left[\tau-\mathbf{1}_{\hat{\varepsilon}_{i}<\hat{Q}_{\varepsilon}(\tau)}\right]^{2}-\frac{1}{n} \sum_{i=1}^{n}\left[\tau-\mathbf{1}_{\varepsilon_{i}<Q_{\varepsilon}(\tau)}\right]^{2} \stackrel{p}{\rightarrow} 0, \\
& \frac{1}{n} \sum_{i=1}^{n} \mathbf{1}_{\hat{\varepsilon}_{i}<\hat{Q}_{\varepsilon}(\tau)} D_{i}(\hat{\theta})-\frac{1}{n} \sum_{i=1}^{n} \mathbf{1}_{\varepsilon_{i}<Q_{\varepsilon}(\tau)} D_{i}(\theta) \stackrel{p}{\rightarrow} 0, \\
& \frac{1}{n} \sum_{i=1}^{n} D_{i}(\hat{\theta}) D_{i}(\hat{\theta})^{T}-\frac{1}{n} \sum_{i=1}^{n} D_{i}(\theta) D_{i}(\theta)^{T} \stackrel{p}{\rightarrow} 0 .
\end{aligned}
$$

Note that $\left|\left[\tau-\mathbf{1}_{\hat{\varepsilon}_{i}<\hat{Q}_{\varepsilon}(\tau)}\right]^{2}-\left[\tau-\mathbf{1}_{\varepsilon_{i}<Q_{\varepsilon}(\tau)}\right]^{2}\right| \leq 2\left|\mathbf{1}_{\hat{\varepsilon}_{i}<\hat{Q}_{\varepsilon}(\tau)}-\mathbf{1}_{\varepsilon_{i}<Q_{\varepsilon}(\tau)}\right|$. Thus,

$$
\left|\frac{1}{n} \sum_{i=1}^{n}\left[\tau-\mathbf{1}_{\hat{\varepsilon}_{i}<\hat{Q}_{\varepsilon}(\tau)}\right]^{2}-\frac{1}{n} \sum_{i=1}^{n}\left[\tau-\mathbf{1}_{\varepsilon_{i}<Q_{\varepsilon}(\tau)}\right]^{2}\right| \leq \frac{2}{n} \sum_{i=1}^{n}\left|\mathbf{1}_{\hat{\varepsilon}_{i}<\hat{Q}_{\varepsilon}(\tau)}-\mathbf{1}_{\varepsilon_{i}<Q_{\varepsilon}(\tau)}\right| \stackrel{p}{\rightarrow} 0,
$$

where the last convergence follows from the proof in Lemma 7. This proves (79). To prove (80), assume without loss of generality that $D\left(\theta, \varepsilon_{i}, \mathbf{X}_{i}\right)$ is scalar-valued. Note that

$$
\begin{aligned}
& \left|\frac{1}{n} \sum_{i=1}^{n} \mathbf{1}_{\hat{\varepsilon}_{i}<\hat{Q}_{\varepsilon}(\tau)} D_{i}(\hat{\theta})-\frac{1}{n} \sum_{i=1}^{n} \mathbf{1}_{\varepsilon_{i}<Q_{\varepsilon}(\tau)} D_{i}(\theta)\right| \\
\leq & \frac{1}{n} \sum_{i=1}^{n} \mathbf{1}_{\hat{\varepsilon}_{i}<\hat{Q}_{\varepsilon}(\tau)}\left|D_{i}(\hat{\theta})-D_{i}(\theta)\right|+\frac{1}{n} \sum_{i=1}^{n}\left|\mathbf{1}_{\hat{\varepsilon}_{i}<\hat{Q}_{\varepsilon}(\tau)}-\mathbf{1}_{\varepsilon_{i}<Q_{\varepsilon}(\tau)}\right|\left|D_{i}(\theta)\right| .
\end{aligned}
$$

By the stochastic continuity of $D_{i}(\vartheta)$ at $\vartheta=\theta$, the argument in the proof of Lemma 1 shows $n^{-1} \sum_{i=1}^{n}\left|D_{i}(\hat{\theta})-D_{i}(\theta)\right| \stackrel{p}{\rightarrow} 0$. Since $D_{0}(\theta) \in \mathcal{L}^{2}$, by the same Dominated Convergence Theorem in the proof of Lemma $7, \mathbb{E}\left[\left|\mathbf{1}_{\hat{\varepsilon}_{0}<\hat{Q}_{\varepsilon}(\tau)}-\mathbf{1}_{\varepsilon_{0}<Q_{\varepsilon}(\tau)}\right|\left|D_{0}(\theta)\right|\right] \rightarrow 0$, which implies (by stationarity) $n^{-1} \sum_{i=1}^{n}\left|\mathbf{1}_{\hat{\varepsilon}_{i}<\hat{Q}_{\varepsilon}(\tau)}-\mathbf{1}_{\varepsilon_{i}<Q_{\varepsilon}(\tau)}\right|\left|D_{i}(\theta)\right| \stackrel{p}{\rightarrow} 0$. Thus, (80) follows from (82). Finally, by the stochastic continuity of $D_{i}(\vartheta) D_{i}(\vartheta)^{T}$ at $\vartheta=\theta$ and Lemma $1,(81)$ holds. $\diamond$

Proof of Theorem 4. First, we show that the asymptotic representation (75) holds uniformly on $\tau \in[\delta, 1-\delta]$. It suffices to show that the quadratic approximation (73) holds uniformly on $[\delta, 1-\delta]$. Recall $I_{1}$ and $I_{2}$ in (69) and (70). In the proof of Theorem 2, it is shown that $I_{1}=\Delta^{2} f_{\varepsilon}\left(Q_{\varepsilon}(\tau)\right) / 2+o_{p}(1)$ and $I_{2}=o_{p}(1)$ for fixed quantile $\tau$. Now we shall prove that they also hold uniformly on $[\delta, 1-\delta]$. By Assumption 4(iv), the continuity of $\dot{J}(0, z)$ on $z \in \mathbb{R}$ implies uniform continuity on compact interval. Thus, 
$\dot{J}\left(0, Q_{\varepsilon}(\tau)+s\right)-\dot{J}\left(0, Q_{\varepsilon}(\tau)\right)=o(1)$ uniformly on $[\delta, 1-\delta]$ and $|s| \leq \Delta / \sqrt{n}$. This shows $I_{2}=o_{p}(1)$ uniformly. Similarly, by the uniform continuity of $f_{\varepsilon}$, (71) holds uniformly. Thus, by Lemma 8 , we conclude that $I_{1}=\Delta^{2} f_{\varepsilon}\left(Q_{\varepsilon}(\tau)\right) / 2+o_{p}(1)$ uniformly on $[\delta, 1-\delta]$.

By the above argument, (75) and hence (76) hold uniformly on $[\delta, 1-\delta]$. To prove the functional CLT, it suffices to prove the tightness and finite-dimensional convergence of the leading term $\dot{G}_{\varepsilon}\left(x, Q_{\varepsilon}(\tau), x\right) n^{-1} \sum_{i=1}^{n} W_{i}(\tau)$ in (76). The tightness follows from two facts: (i) after normalization, the empirical process $n^{-1} \sum_{i=1}^{n} \mathbf{1}_{\varepsilon_{i} \leq Q_{\varepsilon}(\tau)}$ is tight [Chapter 14 in Billingsley (1999)]; and (ii) if $\eta_{n} \Rightarrow \eta$, then for any continuous function $g(\tau)$ the process $\left\{g(\tau) \eta_{n}\right\}_{\tau}$ is tight on $[\delta, 1-\delta]$ [Theorem 7.3 in Billingsley (1999)]. Using (76), the finite-dimensional convergence follows from the Cramér-Wold device and Lemma 5.

Proof of Theorem 6. Recall $\hat{F}(y \mid x)$ in (40). Write $z=H(\theta, y, x)$ and $\hat{z}=H(\hat{\theta}, y, x)$. Since $H(\hat{\theta}, y, x)$ is an increasing function in $y$ (Assumption 1), $H\left(\hat{\theta}, \mathcal{Y}_{1}, x\right) \leq H(\hat{\theta}, y, x) \leq$ $H\left(\hat{\theta}, \mathcal{Y}_{2}, x\right)$ uniformly on $y \in \mathcal{Y}$. Thus, using $\hat{\theta}=\theta+o_{p}(1)$ and the continuity assumption, $|\hat{z}|=O_{p}(1)$ uniformly on $y \in \mathcal{Y}$. Note that $\hat{F}(y \mid x)=\hat{F}_{\varepsilon}(\hat{z})$. By the uniform approximation of $\hat{F}_{\varepsilon}(z)$ in Theorem 1 and the equivalent expression for $\dot{J}(0, z)$ in Lemma 4 , we have

$$
\sup _{y \in \mathcal{Y}}\left|\hat{F}(y \mid x)-\frac{1}{n} \sum_{i=1}^{n} \mathbf{1}_{\varepsilon_{i} \leq \hat{z}}-\dot{J}(0, \hat{z})^{T}(\hat{\theta}-\theta)\right|=o_{p}\left(n^{-1 / 2}\right) .
$$

Furthermore, by $\hat{\theta}-\theta=O_{p}\left(n^{-1 / 2}\right)$ and Assumption 4(iii), we have $\hat{z}-z=O_{p}\left(n^{-1 / 2}\right) L(y, x)=$ $O_{p}\left(n^{-1 / 2}\right)$ uniformly on $y \in \mathcal{Y}$. Therefore, by Lemma 9 ,

$$
\sup _{y \in \mathcal{Y}}\left|\frac{1}{n} \sum_{i=1}^{n} \mathbf{1}_{\varepsilon_{i} \leq \hat{z}}-\frac{1}{n} \sum_{i=1}^{n} \mathbf{1}_{\varepsilon_{i} \leq z}-(\hat{z}-z) f_{\varepsilon}(z)\right|=o_{p}\left(n^{-1 / 2}\right) .
$$

The continuity of $\dot{J}(0, z)$ in Assumption 4(iv) implies the uniform continuity of $\dot{J}(0, z)$ on compact intervals. Thus, $\dot{J}(0, \hat{z})-\dot{J}(0, z)=o_{p}(1)$ uniformly on $y \in \mathcal{Y}$. Combining the latter with (83)-(84) and $\mathbb{E}\left(\mathbf{1}_{\varepsilon_{i} \leq z}\right)=F(y \mid x)$ [see (39)], we obtain the uniform approximation $\hat{F}(y \mid x)-F(y \mid x)=\frac{1}{n} \sum_{i=1}^{n}\left[\mathbf{1}_{\varepsilon_{i} \leq z}-\mathbb{E}\left(\mathbf{1}_{\varepsilon_{i} \leq z}\right)\right]+\dot{J}(0, z)^{T}(\hat{\theta}-\theta)+(\hat{z}-z) f_{\varepsilon}(z)+o_{p}\left(n^{-1 / 2}\right)$.

Hence, by Taylor's expansion $\hat{z}-z=\dot{H}(\theta, y, x)^{T}(\hat{\theta}-\theta)+o_{p}\left(n^{-1 / 2}\right)$, the Bahadur representation for $\hat{\theta}-\theta$ in Assumption 2, and Lemma 4, we can obtain the uniform approximation

$$
\hat{F}(y \mid x)-F(y \mid x)=\frac{1}{n} \sum_{i=1}^{n} V_{i}(y)+o_{p}\left(n^{-1 / 2}\right), \quad y \in \mathcal{Y},
$$


where $V_{i}(y)$ is defined in (43). From (85), the tightness follows from the tightness of the empirical process of $\left\{\varepsilon_{i}\right\}$ and the differentiability of $H(\theta, y, x)$ in $y$, and the finitedimensional convergence follows from the Cramér-Wold device and Lemma 5.

\section{References}

Aït-Sahalia, Y. and A.W. Lo, 2000, Nonparametric risk management and implied risk aversion. Journal of Econometrics 94, 9-51.

Bennett, G., 1962, Probability inequalities for the sum of independent random variables. Journal of the American Statistical Association 57, 33-45.

Billingsley, P., 1999, Convergence of Probability Measures, 2nd edition, John Wiley \& Sons.

Bollerslev, T., 1986, Generalized autoregressive conditional heteroscedasticity. Journal of Econometrics 31, 307-327.

Bühlmann, P., 1997, Sieve bootstrap for time series. Bernoulli 3, 123-148.

Butler, J.S. and B. Schachter, 1998, Estimating Value-at-Risk with a precision measure by combining kernel estimation with historical simulation. Review of Derivatives Research 1, 371-390.

Cai, Z., 2002, Regression quantiles for time series. Econometric Theory 18, 169-192.

Cai Z. and X. Wang, 2008, Nonparametric estimation of conditional VaR and expected shortfall. Journal of Econometrics 147, 120-130.

Chen, S., 2008, Nonparametric estimation of expected shortfall. Journal of Financial Econometrics 6, 87-107.

Chen, S. and C. Tang, 2005, Nonparametric inference of value at risk for dependent financial returns. Journal of Financial Econometrics 3, 227-255.

Chen, X., 2007, Large sample sieve estimation of semi-nonparametric models. In: J.J. Heckman and E.L. Edward (editors), Handbook of Econometrics Vol. 6, Part 2, 5549-5632.

Chernozhukov, V. and L. Umanstev, 2001, Conditional value-at-risk: Aspects of modeling and estimation. Empirical Economics 26, 271-292.

Cosma, A., O. Scaillet and R. von Sachs, 2007, Multivariate wavelet-based shape preserving estimation for dependent observations. Bernoulli 13, 301-329. 
Danielsson, J. and C.G. Vries, de, 2000, Value-at-risk and extreme returns. Annales déconomie et de statistique 60, 236-269.

Dowd, K., 1998, Beyond Value at Risk: The New Science of Risk Management, Wiley, New York.

Duffie, D. and J. Pan, 1997, An overview of value at risk. Journal of Derivatives 4, 7-49.

Engle, R. and S. Manganelli, 2004, CAViaR: Conditional autoregressive value at risk by regression quantile. Journal of Business and Economics Statistics 22, 367-381.

Fan, J. and Q. Yao, 2003, Nonlinear Time Series: Nonparametric and Parametric Methods, Springer, New York.

Fermanian, J.D. and O. Scaillet, 2005, Sensitivity analysis of VaR and Expected Shortfall for portfolios under netting agreements. Journal of Banking and Finance 29, 927-958.

Frey, R. and A.J. McNeil, 2002, VaR and expected shortfall in portfolios of dependent credit risks: Conceptual and practical insights. Journal of Banking and Finance 26, 1317-1334.

Gerlach, R., Z. Lu and H. Huang, 2013, Exponentially smoothing the skewed laplace distribution for value-at-risk forecasting. Journal of Forecasting 32 534-550.

Ghalanos, A., 2014, rugarch: Univariate GARCH models. R package.

Glosten, L.R., R. Jagannathan and D. Runkle, 1993, On the relation between the expected value and the volatility of the nominal excess return on stocks. Journal of Finance 48, 1779-1801.

Gourieroux, X., J.P. Laurent and O. Scaillet, 2000, Sensitivity analysis of Values at Risk. Journal of Empirical Finance 7, 225-245.

Guermat C. and R.D.D. Harris, 2001, Robust conditional variance estimation and valueat-risk. Journal of Risk 4, 25-41.

Hall, P. and Q. Yao, 2003, Inference in ARCH and GARCH models with heavy-tailed errors. Econometrica 71, 285-317.

He, X. and Q.M. Shao, 1996, A general Bahadur representation of M-estimators and its application to linear regression with nonstochastic designs. The Annals of Statistics 24, 2608-2630.

Horowitz, J.L., 1996, Semiparametric estimation of a regression model with an unknown transformation of the dependent variable. Econometrica 64, 103-137.

Horváth, L. and G. Teyssière, 2001, Empirical process of the squared residuals of an arch 
sequence. The Annals of Statistics 29, 445-469.

Hull, J. and A. White, 1998, Value at Risk when daily changes are not normally distributed. Journal of Derivatives 5, 9-19.

Jurečková, J. and B. Procházka, 1994, Regression quantiles and trimmed least squares estimator in nonlinear regression model. Journal of Nonparametric Statistics 3, 201222.

Lahiri, S.N., 2003, Resampling Methods for Dependent Data, Springer Series in Statistics, Springer, New York.

Lee, S. and C.Z. Wei, 1999, On residual empirical processes of stochastic regression models with applications to time series. The Annals of Statistics 27, 237-261.

Li, Q. and J. Racine, 2007, Nonparametric Econometrics, Princeton University Press, Princeton, New Jersey.

Matzkin, R.L., 2003, Nonparametric estimation of nonadditive random functions. Econometrica $71,1339-1375$.

Morgan, J.P., 1996, RiskMetrics Technical Document, 4th edn, New York.

Nelson, D.B., 1991, Conditional heteroskedasticity in asset returns: A new approach. Econometrica 59, 347-370.

Pollard, D., 1991, Asymptotic for least absolute deviation regression estimators. Econometric Theory 7, 186-199.

Portnoy, S. and R. Koenker, 1989, Adaptive L-estimation of Linear Models. The Annals of Statistics 17, 362-381.

Scaillet, O., 2003, The origin and development of VaR. In Modern Risk Management: A History, 15th Anniversary of Risk Magazine, Risk Publications, London, 151-158.

Scaillet, O., 2004, Nonparametric estimation and sensitivity analysis of expected shortfall. Mathematical Finance 14, 115-129.

Scaillet, O., 2005, Nonparametric estimation of conditional expected shortfall. Revue Assurances et Gestion des Risques/Insurance and Risk Management Journal 74, 639660.

Wu, W.B., K. Yu and G. Mitra, 2007, Kernel conditional quantile estimation for stationary processes with application to conditional Value-at-Risk. Journal of Financial Econometrics 6, 253-270.

Wu, W.B. and X. Shao, 2004, Limit theorems for iterated random functions. Journal of 
Applied Probability 41, 425-436.

Wu, W.B. and Z. Zhao, 2008, Moderate deviations for stationary processes. Statistica Sinica 18, 769-782.

Yu, K. and M.C. Jones, 1998, Local linear quantile regression. Journal of the American Statistical Association 93, 228-237.

Zakoian, J.M., 1994, Threshold heteroskedastic models. Journal of Economic Dynamics and Control 18, 931-955.

Zhao, Z., 2010, Density estimation for nonlinear parametric models with conditional heteroscedasticity. Journal of Econometrics 155, 71-82.

Zhao, Z. and Z. Xiao, 2014, Efficient regressions via optimally combining quantile information. Econometric Theory 30, 1272-1314.

Table 1: RMISE [see (46)] of the proposed semiparametric estimate of $\operatorname{CVaR}(1-\tau \mid x)=Q(\tau \mid x)$ relative to the nonparametric method in (3) with theoretical optimal bandwidth, at different quantiles $\tau$. Numbers $\geq 1$ indicate better performance of the proposed method.

\begin{tabular}{|c|c|c|c|c|c|c|c|c|c|c|c|c|c|c|}
\hline & \multirow[b]{2}{*}{ noise } & \multicolumn{13}{|c|}{ Quantile $\tau$ in $\operatorname{CVaR}(1-\tau \mid x)=Q(\tau \mid x)$} \\
\hline & & $1 \%$ & $5 \%$ & $10 \%$ & $20 \%$ & $30 \%$ & $40 \%$ & $50 \%$ & $60 \%$ & $70 \%$ & $80 \%$ & $90 \%$ & $95 \%$ & $99 \%$ \\
\hline \multirow[t]{2}{*}{ Model 1} & $N(0,1)$ & 2.45 & 2.09 & 1.62 & 1.54 & 1.47 & 1.42 & 1.35 & 1.32 & 1.34 & 1.38 & 1.72 & 2.03 & 2.81 \\
\hline & $t_{3} / \sqrt{3}$ & 3.01 & 2.39 & 2.11 & 1.50 & 1.14 & 0.96 & 0.94 & 0.98 & 1.05 & 1.34 & 1.80 & 2.18 & 3.38 \\
\hline \multirow[t]{2}{*}{ Model 2} & $N(0,1)$ & 3.54 & 2.92 & 2.14 & 1.67 & 1.50 & 1.43 & 1.39 & 1.38 & 1.46 & 1.69 & 2.19 & 2.61 & 3.61 \\
\hline & $t_{3} / \sqrt{3}$ & 5.16 & 3.22 & 2.25 & 1.40 & 1.09 & 1.01 & 0.97 & 0.99 & 1.15 & 1.45 & 2.67 & 3.67 & 4.03 \\
\hline \multirow[t]{2}{*}{ Model 3} & $N(0,1)$ & 2.58 & 2.01 & 1.80 & 1.48 & 1.27 & 1.30 & 1.37 & 1.54 & 1.86 & 2.11 & 2.15 & 2.32 & 2.93 \\
\hline & $t_{3} / \sqrt{3}$ & 1.85 & 1.01 & 0.84 & 0.80 & 0.86 & 0.87 & 0.88 & 0.91 & 0.90 & 1.00 & 1.17 & 1.36 & 2.24 \\
\hline \multirow[t]{2}{*}{ Model 4} & $N(0,1)$ & 3.65 & 2.61 & 2.28 & 2.15 & 1.79 & 1.45 & 1.35 & 1.46 & 1.71 & 1.97 & 2.19 & 2.60 & 3.61 \\
\hline & $t_{3} / \sqrt{3}$ & 2.71 & 1.13 & 1.01 & 0.95 & 0.87 & 0.81 & 0.78 & 0.77 & 0.86 & 1.00 & 1.02 & 1.17 & 3.09 \\
\hline
\end{tabular}


Table 2: Empirical coverage rate for GARCH models 5-7 of the proposed semiparametric estimate of $\operatorname{CVaR}(1-\tau \mid x)=Q(\tau \mid x)$ at different quantiles $\tau$.

\begin{tabular}{|c|c|c|c|c|c|c|c|c|c|c|c|c|c|c|c|}
\hline & \multirow[b]{2}{*}{ noise } & \multirow[b]{2}{*}{$n$} & \multicolumn{13}{|c|}{ Quantile $\tau$ in $\operatorname{CVaR}(1-\tau \mid x)=Q(\tau \mid x)$} \\
\hline & & & $1 \%$ & $5 \%$ & $10 \%$ & $20 \%$ & $30 \%$ & $40 \%$ & $50 \%$ & $60 \%$ & $70 \%$ & $80 \%$ & $90 \%$ & $95 \%$ & $99 \%$ \\
\hline \multirow[t]{4}{*}{ Model 5} & $N(0,1)$ & $n=200$ & 0.014 & 0.046 & 0.094 & 0.195 & 0.293 & 0.388 & 0.498 & 0.610 & 0.713 & 0.800 & 0.900 & 0.950 & 0.983 \\
\hline & & $n=500$ & 0.012 & 0.049 & 0.098 & 0.198 & 0.303 & 0.399 & 0.497 & 0.597 & 0.692 & 0.777 & 0.884 & 0.938 & 0.983 \\
\hline & $t_{3} / \sqrt{3}$ & $n=200$ & 0.017 & 0.045 & 0.087 & 0.171 & 0.273 & 0.386 & 0.489 & 0.590 & 0.685 & 0.804 & 0.904 & 0.946 & 0.984 \\
\hline & & $n=500$ & 0.012 & 0.056 & 0.111 & 0.219 & 0.314 & 0.405 & 0.503 & 0.604 & 0.698 & 0.811 & 0.896 & 0.942 & 0.992 \\
\hline \multirow[t]{4}{*}{ Model 6} & $N(0,1)$ & $n=200$ & 0.019 & 0.055 & 0.103 & 0.202 & 0.301 & 0.399 & 0.502 & 0.605 & 0.703 & 0.801 & 0.898 & 0.948 & 0.982 \\
\hline & & $n=500$ & 0.012 & 0.051 & 0.101 & 0.196 & 0.299 & 0.404 & 0.506 & 0.604 & 0.705 & 0.805 & 0.904 & 0.954 & 0.990 \\
\hline & $t_{3} / \sqrt{3}$ & $n=200$ & 0.023 & 0.063 & 0.110 & 0.208 & 0.302 & 0.408 & 0.503 & 0.601 & 0.695 & 0.794 & 0.893 & 0.938 & 0.978 \\
\hline & & $n=500$ & 0.012 & 0.048 & 0.096 & 0.200 & 0.302 & 0.402 & 0.504 & 0.600 & 0.711 & 0.808 & 0.904 & 0.955 & 0.988 \\
\hline \multirow[t]{4}{*}{ Model 7} & $N(0,1)$ & $n=200$ & 0.019 & 0.061 & 0.111 & 0.207 & 0.304 & 0.409 & 0.505 & 0.601 & 0.698 & 0.801 & 0.902 & 0.949 & 0.982 \\
\hline & & $n=500$ & 0.011 & 0.056 & 0.106 & 0.207 & 0.303 & 0.402 & 0.497 & 0.597 & 0.698 & 0.797 & 0.894 & 0.945 & 0.985 \\
\hline & $t_{3} / \sqrt{3}$ & $n=200$ & 0.019 & 0.062 & 0.112 & 0.213 & 0.309 & 0.414 & 0.517 & 0.609 & 0.707 & 0.802 & 0.899 & 0.953 & 0.986 \\
\hline & & $n=500$ & 0.015 & 0.053 & 0.094 & 0.189 & 0.283 & 0.385 & 0.481 & 0.581 & 0.683 & 0.790 & 0.885 & 0.939 & 0.986 \\
\hline
\end{tabular}

Table 3: RMSE [see (47)] of the proposed nonparametric distribution method relative to three parametricdistribution (Normal, Student- $t$, and asymmetric-Laplace (ALD)) based competitors in the presence of different noise distributions: $N(0,1), t_{3} / \sqrt{3}$, standard Laplace $/ \sqrt{2}$, Normal mixture $0.5 N(0,0.5)+$ $0.5 N(0,1.5)$, and standard exponential minus 1 . Numbers $\geq 1$ indicate better performance of the proposed nonparametric distribution method. For convenience, numbers $\geq 100$ are marked as $\infty$.

\begin{tabular}{|c|c|c|c|c|c|c|c|c|c|c|c|c|c|c|}
\hline \multirow[b]{2}{*}{ noise } & \multirow[b]{2}{*}{ Method } & \multicolumn{13}{|c|}{ Quantile $\tau$ in $\operatorname{CVaR}(1-\tau \mid x)=Q(\tau \mid x)$} \\
\hline & & $1 \%$ & $5 \%$ & $10 \%$ & $20 \%$ & $30 \%$ & $40 \%$ & $50 \%$ & $60 \%$ & $70 \%$ & $80 \%$ & $90 \%$ & $95 \%$ & $99 \%$ \\
\hline \multirow[t]{3}{*}{$N(0,1)$} & Normal & 0.27 & 0.55 & 0.62 & 0.66 & 0.68 & 0.66 & 0.66 & 0.66 & 0.68 & 0.65 & 0.63 & 0.53 & 0.27 \\
\hline & Student- $t$ & 0.36 & 0.54 & 0.68 & 0.75 & 0.72 & 0.67 & 0.63 & 0.66 & 0.69 & 0.73 & 0.67 & 0.53 & 0.36 \\
\hline & ALD & 45.66 & 9.15 & 0.90 & 6.68 & 8.35 & 3.84 & 0.16 & 3.80 & 8.21 & 6.58 & 0.91 & 9.24 & 46.80 \\
\hline \multirow[t]{3}{*}{$t_{3} / \sqrt{3}$} & Normal & 2.50 & 16.72 & 45.77 & 70.69 & 47.36 & 14.17 & 1.56 & 14.18 & 46.97 & 70.20 & 47.17 & 16.28 & 2.62 \\
\hline & Student- $t$ & 0.34 & 0.36 & 0.48 & 0.71 & 0.80 & 0.81 & 0.81 & 0.82 & 0.81 & 0.72 & 0.50 & 0.37 & 0.34 \\
\hline & ALD & 0.35 & 2.21 & 3.34 & 1.50 & 1.10 & 1.08 & 0.42 & 1.07 & 1.07 & 1.45 & 3.40 & 2.24 & 0.35 \\
\hline \multirow[t]{3}{*}{ Laplace $/ \sqrt{2}$} & Normal & 4.19 & 0.49 & 4.94 & 19.24 & 23.36 & 12.29 & 1.83 & 12.19 & 22.71 & 18.35 & 4.96 & 0.52 & 4.22 \\
\hline & Student- $t$ & 0.81 & 1.05 & 1.01 & 0.67 & 1.68 & 2.05 & 1.21 & 2.08 & 1.67 & 0.66 & 1.02 & 1.09 & 0.82 \\
\hline & ALD & 0.28 & 0.55 & 0.66 & 0.67 & 0.61 & 0.54 & 0.49 & 0.54 & 0.61 & 0.66 & 0.68 & 0.59 & 0.29 \\
\hline \multirow[t]{3}{*}{ Normal mixture } & Normal & 1.77 & 0.44 & 1.24 & 2.95 & 2.31 & 1.22 & 0.79 & 1.31 & 2.56 & 3.21 & 1.51 & 0.43 & 1.74 \\
\hline & Student- $t$ & 0.40 & 0.49 & 0.59 & 0.82 & 0.83 & 0.77 & 0.75 & 0.76 & 0.83 & 0.82 & 0.59 & 0.49 & 0.40 \\
\hline & ALD & 10.93 & 3.40 & 0.86 & 3.11 & 4.89 & 2.67 & 0.20 & 2.62 & 4.89 & 3.12 & 0.86 & 3.44 & 10.77 \\
\hline \multirow[t]{3}{*}{ Exponential -1} & Normal & $\infty$ & $\infty$ & $\infty$ & 19.35 & 34.41 & 84.45 & 95.26 & 76.85 & 46.08 & 15.23 & 0.81 & 7.17 & 16.84 \\
\hline & Student- $t$ & $\infty$ & $\infty$ & $\infty$ & 36.11 & 2.11 & 4.53 & 2.27 & 2.09 & 9.13 & 24.43 & 38.29 & 35.09 & 7.82 \\
\hline & ALD & $\infty$ & $\infty$ & $\infty$ & 43.73 & $\infty$ & $\infty$ & $\infty$ & 41.36 & 13.43 & 1.67 & 2.09 & 5.36 & 5.59 \\
\hline
\end{tabular}


Table 4: RMSE [see (47)] of the proposed nonparametric distribution method relative to three parametricdistribution (Normal, Student- $t$, and asymmetric-Laplace (ALD)) based competitors in the presence of model mis-specification described in Section 6.4. The same five noise distributions in Table 3 are used. Numbers $\geq 1$ indicate better performance of the proposed nonparametric distribution method.

\begin{tabular}{|c|c|c|c|c|c|c|c|c|c|c|c|c|c|}
\hline \multirow[b]{3}{*}{ noise } & \multirow[b]{3}{*}{ Method } & \multicolumn{12}{|c|}{ Quantile $\tau$ in $\operatorname{CVaR}(1-\tau \mid x)=Q(\tau \mid x)$} \\
\hline & & \multicolumn{6}{|c|}{ Deviation parameter $\gamma=0.2$} & \multicolumn{6}{|c|}{ Deviation parameter $\gamma=0.4$} \\
\hline & & $60 \%$ & $70 \%$ & $80 \%$ & $90 \%$ & $95 \%$ & $99 \%$ & $60 \%$ & $70 \%$ & $80 \%$ & $90 \%$ & $95 \%$ & $99 \%$ \\
\hline \multirow[t]{3}{*}{$N(0,1)$} & Normal & 0.70 & 0.9 & 0.94 & 0.90 & 0.97 & 0.94 & 0.86 & 0.94 & 0.97 & 1.01 & 0.90 & 0.85 \\
\hline & Student- $t$ & 0.71 & 0.9 & 0.94 & 0.90 & 0.97 & 1.01 & 0.86 & 0.95 & 0.97 & 1.01 & 0.90 & 0.90 \\
\hline & ALD & 2.81 & 4.0 & 2.19 & 0.95 & 2.90 & 14.64 & 2.94 & 3.09 & 1.82 & 1.03 & 1.66 & 6.73 \\
\hline \multirow[t]{3}{*}{$t_{3} / \sqrt{3}$} & Normal & 10.41 & 15.02 & 11.87 & 7.35 & 3.85 & 0.56 & 5.56 & 7.81 & 7.42 & 4.65 & 2.37 & 1.19 \\
\hline & Student- $t$ & 1.00 & 1.09 & 0.91 & 0.87 & 0.90 & 0.64 & 0.81 & 1.04 & 1.09 & 0.98 & 0.79 & 1.24 \\
\hline & ALD & 0.70 & 1.12 & 1.47 & 1.94 & 1.82 & 0.42 & 0.91 & 1.33 & 1.58 & 1.48 & 1.05 & 0.84 \\
\hline \multirow[t]{3}{*}{ Laplace $/ \sqrt{2}$} & Normal & 11.79 & 12.82 & 8.39 & 3.52 & 1.39 & 1.27 & 10.94 & 9.66 & 6.05 & 2.00 & 0.91 & 1.63 \\
\hline & Student- $t$ & 1.96 & 1.44 & 0.99 & 1.10 & 1.16 & 1.39 & 1.75 & 1.22 & 1.01 & 0.98 & 0.89 & 1.13 \\
\hline & ALD & 0.74 & 0.71 & 0.85 & 1.10 & 1.10 & 0.64 & 0.72 & 0.85 & 1.01 & 0.96 & 0.87 & 0.83 \\
\hline \multirow[t]{3}{*}{ Normal mixture } & Normal & 1.08 & 1.48 & 1.55 & 1.12 & 0.84 & 1.26 & 1.11 & 1.65 & 1.52 & 1.29 & 0.83 & 0.76 \\
\hline & Student- $t$ & 0.77 & 0.88 & 0.98 & 0.95 & 0.86 & 0.79 & 0.80 & 1.09 & 1.04 & 1.05 & 0.79 & 0.74 \\
\hline & ALD & 2.12 & 2.50 & 1.56 & 0.99 & 1.49 & 4.21 & 1.07 & 1.39 & 1.02 & 1.16 & 1.35 & 2.75 \\
\hline \multirow[t]{3}{*}{ Exponential -1} & Normal & 70.61 & 31.77 & 6.17 & 0.79 & 2.22 & 7.55 & 78.01 & 21.34 & 3.33 & 1.05 & 1.61 & 3.09 \\
\hline & Student- $t$ & 1.89 & 6.09 & 9.00 & 10.02 & 8.67 & 3.74 & 1.67 & 4.29 & 4.87 & 5.11 & 4.19 & 1.86 \\
\hline & ALD & 37.92 & 9.47 & 1.15 & 1.14 & 1.87 & 2.97 & 41.89 & 6.42 & 1.05 & 1.22 & 1.46 & 1.60 \\
\hline
\end{tabular}


Table 5: Empirical coverage probability of confidence intervals (CI) for CVaR $(0.05 \mid x)$.

(Model 1) CVaR $(0.05 \mid x): x$ at different percentiles of covariates $\left\{Y_{i-1}\right\}$

\begin{tabular}{|c|c|c|c|c|c|c|c|c|c|c|c|c|c|}
\hline \multirow[b]{2}{*}{ noise } & \multirow{2}{*}{$\begin{array}{l}\text { CI level } \\
1-\alpha\end{array}$} & \multicolumn{6}{|c|}{$\begin{array}{c}\text { Asymptotic CI for CVaR }(0.05 \mid x) \\
x \text { at different percentiles }\end{array}$} & \multicolumn{6}{|c|}{$\begin{array}{c}\text { Bootstrap CI for CVaR }(0.05 \mid x) \\
x \text { at different percentiles }\end{array}$} \\
\hline & & 25 th & 50 th & 75 th & 90th & 95th & 99th & 25 th & 50th & 75 th & 90th & 95 th & 99th \\
\hline \multirow[t]{3}{*}{$N(0,1)$} & $90 \%$ & 85.8 & 83.3 & 82.5 & 82.6 & 84.4 & 85.2 & 90.4 & 88.7 & 88.5 & 87.8 & 88.2 & 88.2 \\
\hline & $95 \%$ & 91.7 & 89.3 & 88.7 & 89.2 & 90.0 & 90.7 & 94.6 & 94.1 & 93.9 & 94.1 & 94.9 & 95.1 \\
\hline & $99 \%$ & 96.0 & 95.7 & 95.5 & 96.2 & 96.8 & 97.6 & 98.2 & 97.8 & 97.6 & 98.7 & 98.6 & 98.9 \\
\hline \multirow[t]{3}{*}{$t_{3} / \sqrt{3}$} & $90 \%$ & 83.2 & 82.2 & 82.8 & 83.2 & 83.2 & 86.2 & 90.0 & 89.8 & 90.4 & 91.3 & 92.0 & 92.2 \\
\hline & $95 \%$ & 87.6 & 88.0 & 87.6 & 88.6 & 90.0 & 91.4 & 94.1 & 94.4 & 95.2 & 94.8 & 95.8 & 95.6 \\
\hline & $99 \%$ & 94.2 & 94.2 & 94.6 & 96.0 & 96.6 & 97.2 & 98.8 & 98.4 & 98.4 & 98.6 & 98.8 & 98.8 \\
\hline
\end{tabular}

(Model 2) $\operatorname{CVaR}\left(0.05 \mid\left(x_{1}, x_{2}\right)\right): x_{1}$ at different percentiles of $\left\{Y_{i-1}\right\}, x_{2}$ at median of $\left\{Y_{i-2}\right\}$

\begin{tabular}{|c|c|c|c|c|c|c|c|c|c|c|c|c|c|}
\hline \multirow[b]{2}{*}{ noise } & \multirow{2}{*}{$\begin{array}{c}\text { CI level } \\
1-\alpha\end{array}$} & \multicolumn{6}{|c|}{$\begin{array}{l}\text { Asymptotic CI for } \operatorname{CVaR}\left(0.05 \mid\left(x_{1}, x_{2}\right)\right) \\
x_{1} \text { at different percentiles, } x_{2} \text { at median }\end{array}$} & \multicolumn{6}{|c|}{$\begin{array}{l}\text { Bootstrap CI for } \operatorname{CVaR}\left(0.05 \mid\left(x_{1}, x_{2}\right)\right) \\
x_{1} \text { at different percentiles, } x_{2} \text { at median }\end{array}$} \\
\hline & & 25 th & 50 th & 75 th & 90th & 95th & 99th & 25 th & 50 th & 75th & 90th & 95th & 99th \\
\hline \multirow[t]{3}{*}{$N(0,1)$} & $90 \%$ & 90.3 & 89.6 & 88.7 & 88.2 & 88.2 & 88.7 & 91.8 & 91.7 & 91.4 & 91.3 & 91.9 & 91.0 \\
\hline & $95 \%$ & 94.7 & 93.5 & 92.6 & 93.1 & 93.3 & 94.4 & 95.9 & 95.8 & 96.3 & 95.6 & 95.6 & 95.8 \\
\hline & $99 \%$ & 98.6 & 97.2 & 96.5 & 97.5 & 98.1 & 97.9 & 99.3 & 98.8 & 98.1 & 98.1 & 98.6 & 98.6 \\
\hline \multirow[t]{3}{*}{$t_{3} / \sqrt{3}$} & $90 \%$ & 83.2 & 82.8 & 82.8 & 84.4 & 85.0 & 85.6 & 87.4 & 87.1 & 87.0 & 88.4 & 90.2 & 89.8 \\
\hline & $95 \%$ & 88.2 & 87.0 & 86.6 & 89.0 & 89.2 & 91.4 & 93.2 & 93.8 & 93.6 & 92.8 & 92.4 & 93.6 \\
\hline & $99 \%$ & 94.2 & 93.0 & 92.6 & 93.2 & 94.4 & 96.6 & 98.0 & 97.0 & 97.4 & 97.6 & 98.2 & 98.8 \\
\hline
\end{tabular}

(Model 3) $\operatorname{CVaR}(0.05 \mid x): x$ at different percentiles of $\left\{Y_{i-1}\right\}$

\begin{tabular}{|c|c|c|c|c|c|c|c|c|c|c|c|c|c|}
\hline \multirow[b]{2}{*}{ noise } & \multirow{2}{*}{$\begin{array}{l}\text { CI level } \\
1-\alpha\end{array}$} & \multicolumn{6}{|c|}{$\begin{array}{c}\text { Asymptotic CI for CVaR }(0.05 \mid x) \\
x \text { at different percentiles }\end{array}$} & \multicolumn{6}{|c|}{$\begin{array}{c}\text { Bootstrap CI for } \operatorname{CVaR}(0.05 \mid x) \\
x \text { at different percentiles }\end{array}$} \\
\hline & & 25 th & 50th & 75 th & 90th & 95th & 99th & 25 th & 50th & 75 th & 90th & 95 th & 99th \\
\hline \multirow[t]{3}{*}{$N(0,1)$} & $90 \%$ & 100 & 100 & 99.8 & 97.7 & 94.2 & 85.0 & 89.0 & 87.3 & 88.3 & 89.1 & 88.3 & 89.0 \\
\hline & $95 \%$ & 100 & 100 & 100 & 99.8 & 98.5 & 91.7 & 93.6 & 93.6 & 94.2 & 93.3 & 93.6 & 95.0 \\
\hline & $99 \%$ & 100 & 100 & 100 & 100 & 100 & 97.7 & 97.7 & 98.8 & 98.3 & 98.1 & 98.3 & 98.8 \\
\hline \multirow[t]{3}{*}{$t_{3} / \sqrt{3}$} & $90 \%$ & 99.3 & 99.3 & 98.3 & 96.3 & 94.1 & 86.6 & 85.6 & 90.7 & 90.2 & 86.3 & 86.1 & 85.9 \\
\hline & $95 \%$ & 99.8 & 99.8 & 99.0 & 98.0 & 97.6 & 91.0 & 91.4 & 94.1 & 94.2 & 91.2 & 89.7 & 89.9 \\
\hline & $99 \%$ & 100 & 100 & 99.5 & 99.0 & 98.5 & 95.8 & 97.8 & 98.0 & 97.3 & 97.3 & 96.8 & 96.1 \\
\hline
\end{tabular}

(Model 4) $\operatorname{CVaR}\left(0.05 \mid\left(x_{1}, x_{2}\right)\right): x_{1}$ at different percentiles $\left\{Y_{i-1}\right\}, x_{2}$ at median of $\left\{U_{i}\right\}$

\begin{tabular}{|c|c|c|c|c|c|c|c|c|c|c|c|c|c|}
\hline \multirow[b]{2}{*}{ noise } & \multirow{2}{*}{$\begin{array}{c}\text { CI level } \\
1-\alpha\end{array}$} & \multicolumn{6}{|c|}{$\begin{array}{l}\text { Asymptotic CI for CVaR }\left(0.05 \mid\left(x_{1}, x_{2}\right)\right) \\
x_{1} \text { at different percentiles, } x_{2} \text { at median }\end{array}$} & \multicolumn{6}{|c|}{$\begin{array}{c}\text { Bootstrap CI for } \operatorname{CVaR}\left(0.05 \mid\left(x_{1}, x_{2}\right)\right) \\
x_{1} \text { at different percentiles, } x_{2} \text { at median }\end{array}$} \\
\hline & & 25 th & 50 th & 75 th & 90th & 95th & 99th & 25 th & 50 th & 75 th & 90th & 95th & 99th \\
\hline \multirow[t]{3}{*}{$N(0,1)$} & $90 \%$ & 100 & 100 & 99.7 & 99.2 & 98.3 & 95.7 & 88.8 & 89.6 & 88.5 & 87.5 & 87.3 & 88.2 \\
\hline & $95 \%$ & 100 & 100 & 99.8 & 99.7 & 100 & 98.8 & 95.3 & 94.1 & 94.4 & 92.8 & 93.1 & 93.2 \\
\hline & $99 \%$ & 100 & 100 & 100 & 100 & 100 & 100 & 99.2 & 98.9 & 98.2 & 97.2 & 96.0 & 96.0 \\
\hline \multirow[t]{3}{*}{$t_{3} / \sqrt{3}$} & $90 \%$ & 100 & 100 & 100 & 98.5 & 95.5 & 86.2 & 88.2 & 89.1 & 89.9 & 83.4 & 80.3 & 75.7 \\
\hline & $95 \%$ & 100 & 100 & 100 & 99.3 & 97.2 & 89.9 & 93.7 & 95.9 & 95.1 & 91.7 & 87.4 & 83.8 \\
\hline & $99 \%$ & 100 & 100 & 100 & 99.8 & 99.4 & 95.5 & 98.5 & 98.9 & 98.3 & 97.4 & 95.7 & 92.0 \\
\hline
\end{tabular}

